\title{
ON CLUSTER ALGEBRAS ARISING FROM UNPUNCTURED SURFACES II
}

\author{
RALF SCHIFFLER
}

\begin{abstract}
We study cluster algebras with principal and arbitrary coefficient systems that are associated to unpunctured surfaces. We give a direct formula for the Laurent polynomial expansion of cluster variables in these cluster algebras in terms of certain paths on a triangulation of the surface. As an immediate consequence, we prove the positivity conjecture of Fomin and Zelevinsky for these cluster algebras.

Furthermore, we obtain direct formulas for $F$-polynomials and $g$-vectors and show that $F$-polynomials have constant term equal to 1 . As an application, we compute the Euler-Poincaré characteristic of quiver Grassmannians in Dynkin type $A$ and affine Dynkin type $\tilde{A}$.
\end{abstract}

\section{Introduction}

Cluster algebras have been introduced by Fomin and Zelevinsky in [FZ1] in order to create an algebraic framework for total positivity and canonical bases in semisimple algebraic groups. Today the theory of cluster algebras is connected to many different areas of mathematics, for example, representation theory of finite dimensional algebras, Lie theory, Poisson geometry and Teichmüller Theory.

Cluster algebras are commutative algebras with a distinguished set of generators, the cluster variables. The set of all cluster variables is constructed recursively from an initial set of $n$ cluster variables using so-called mutations. Every mutation defines a new cluster variable as a rational function of the cluster variables constructed previously; thus recursively, every cluster variable is a certain rational function in the initial $n$ cluster variables. Fomin and Zelevinsky have shown in [FZ1] that these rational functions are actually Laurent polynomials.

The first main result of this paper is a direct formula for these Laurent polynomials for the class of cluster algebras that are associated to oriented unpunctured Riemann surfaces with boundary. Let us point out that this formula holds for arbitrary coefficient systems.

In order to be more precise, we need some notation. The cluster variables are grouped into sets of constant cardinality $n$, the clusters. The integer $n$ is called the rank of the cluster algebra. The cluster algebra is determined by its initial seed which consists of a cluster $\mathbf{x}=\left\{x_{1}, x_{2}, \ldots, x_{n}\right\}$ together with a coefficient tuple $\mathbf{y}=\left\{y_{1}, y_{2}, \ldots, y_{n}\right\}$, and a skew-symmetrizable $n \times n$ integer matrix $B=\left(b_{i j}\right)$. The coefficients $y_{1}, y_{2}, \ldots, y_{n}$ are taken in a torsion free abelian group $\mathbb{P}$. The mutation in direction $k$ defines a new cluster $\mathbf{x}^{\prime}=\mathbf{x} \backslash\left\{x_{k}\right\} \cup\left\{x_{k}^{\prime}\right\}$, where

Date: October 31, 2018.

The author is supported by the NSF grant DMS-0700358 and by the University of Connecticut. 


$$
x_{k}^{\prime}=\frac{1}{x_{k}}\left(y^{+} \prod_{b_{k i}>0} x_{i}^{b_{k i}}+y^{-} \prod_{b_{k i}<0} x_{i}^{-b_{k i}}\right),
$$

where $y^{+}, y^{-}$are certain monomials in the $y_{1}, y_{2}, \ldots, y_{n}$; the precise definition is given in section 2.1. Mutations also transform the coefficient tuple $\mathbf{y}$ and the matrix $B$.

If $u$ is any cluster variable, thus $u$ is obtained from the initial cluster $\left\{x_{1}, \ldots, x_{n}\right\}$ by a sequence of mutations, then, by [FZ1], $u$ can be written as a Laurent polynomial in the variables $x_{1}, x_{2}, \ldots, x_{n}$, that is,

$$
u=\frac{f\left(x_{1}, x_{2}, \ldots, x_{n}\right)}{\prod_{i=1}^{n} x_{i}^{d_{i}}},
$$

where $f$ is a polynomial with coefficients in the group ring $\mathbb{Z P}$ of the coefficient group $\mathbb{P}$.

Inspired by the work of Fock and Goncharov [FG1, FG2, FG3 and Gekhtman, Shapiro and Vainshtein GSV1, GSV2 which discovered cluster structures in the context of Teichmüller theory, Fomin, Shapiro and Thurston [FST] and [FT] initiated a systematic study of the cluster algebras arising from oriented Riemann surfaces with boundary and marked points, a vast class of cluster algebras.

In this approach, cluster variables correspond to isotopyclasses of certain curves in the surface that are called arcs. Clusters are in bijection with triangulations, which are maximal sets of non-crossing arcs, see section 2.3 for precise definitions. The cluster algebras arising from surfaces all share the property that the number of different matrices that can be obtained from the initial matrix by sequences of mutations is finite [FST, Corollary 12.2]. Cluster algebras with this property are called mutation finite. While surfaces provide a large class of examples, not all mutation finite cluster algebras are given by surfaces, for example the Dynkin types $E_{6}, E_{7}, E_{8}$ and the affine Dynkin types $\tilde{E}_{6}, \tilde{E}_{7}, \tilde{E}_{8}$ are not.

Assume from now on that the surface has no punctures, which means that all marked points lie on the boundary of the surface. The cluster algebras arising from these unpunctured surfaces form a three parameter family, the parameters being the genus of the surface, the number of boundary components, and the number of marked points. Fix an arbitrary triangulation $T$ and denote the corresponding cluster by $\mathbf{x}_{T}$. Let $x_{\gamma}$ be an arbitrary cluster variable, where $\gamma$ is an arc in the surface. We know by equation (2) that $x_{\gamma}$ is a Laurent polynomial in $\mathbf{x}_{T}$.

Following [S, [ST, $\mathrm{MS}$, we introduce complete $(T, \gamma)$-paths as paths given by concatenation of arcs of the triangulation such that the arcs in even positions are precisely the arcs of $T$ that are crossed by $\gamma$ in order, and moreover, between any two such crossings, the complete $(T, \gamma)$-path is homotopic to $\gamma$.

Suppose for a moment that the cluster algebra has principal coefficients. Then the Laurent polynomial of equation (2) can be written simply as

$$
x_{\gamma}=\sum_{\alpha} x(\alpha) y(\alpha),
$$

where the sum is over all complete $(T, \gamma)$-paths $\alpha$, where $x(\alpha)$ is the quotient of the cluster variables associated to the odd $\operatorname{arcs}$ in $\alpha$ by the cluster variables associated to the even $\operatorname{arcs}$ in $\alpha$, and where $y(\alpha)$ is a product of elements of the initial coefficient tuple that is depending on the orientation of the path $\alpha$, see Theorem 3.2 
Using results from FZ2, we deduce a formula for the Laurent polynomials in cluster algebras with arbitrary coefficients, see section 7 . As an immediate corollary, we prove, for cluster algebras arising from unpunctured surfaces, that Fomin and Zelevinsky's positivity conjecture of 2002 holds, which states that the coefficients in the Laurent polynomials are non-negative integer linear combinations of elements of the coefficient group. This result was shown for finite type cluster algebras in FZ2; the only finite type arising from unpunctured surfaces is the Dynkin type $A$, where the corresponding surface is a polygon. In $[\mathrm{CK}$ and $[\mathrm{CR}$ the conjecture was shown in the case where the cluster algebra has trivial coefficients and the initial seed is acyclic.

Our formula for the Laurent polynomials in Theorem 3.2 is a continuation of [ST, where a similar formula was shown for cluster algebras with a very limited coefficient system that was associated to the boundary of the surface. The very special case where the surface is a polygon and coefficients arise from the boundary was covered in $[\underline{\mathrm{S}}]$ and also in unpublished work $[\mathrm{CP}, \mathrm{FZ3}]$. Our two papers $[\underline{\mathrm{S}}, \mathbf{S T}]$ used (ordinary) $(T, \gamma)$-paths; the complete $(T, \gamma)$-paths that we use here where introduced in $\mathrm{MS}$. The paper $\mathrm{MS}$ gives a new parametrization of the formulas in terms of perfect matchings.

The proof of Theorem 3.2 uses the theory of covering spaces and involves cluster algebras with a specially designed coefficient system which combines principal coefficients and boundary coefficients.

A second main result of this article consists in explicit formulas for the $F$ polynomials and the $g$-vectors defined in FZ2, see Theorem 6.1 and Theorem 6.5. The formula for the $F$-polynomial yields a proof for a conjecture of [FZ2] stating that $F$-polynomials have constant term 1.

Theorem 3.2 has interesting intersections with work of other people. In CCS2, the authors obtained a formula for the denominators of the cluster expansion in types $A, D$ and $E$, see also BMR2. In CC, CK, CK2 an expansion formula was given in the case where the cluster algebra is acyclic and the cluster lies in an acyclic seed. Palu generalized this formula to arbitrary clusters in an acyclic cluster algebra $\mathrm{Pa}$. These formulas use the cluster category introduced in BMRRT, and in CCS for type $A$, and do not give information about the coefficients.

Recently, Fu and Keller generalized this formula further to cluster algebras with principal coefficients that admit a categorification by a 2-Calabi-Yau category [FK], and, combining results of $\mathrm{A}$ and $\mathrm{ABCP}, \mathrm{LF}$, such a categorification exists in the case of cluster algebras associated to unpunctured surfaces. We thank Bernhard Keller for pointing out this last fact to us. The formula of Keller and Fu involve the Euler-Poincaré characteristic of quiver Grassmannians. Comparing it to our result, we derive a formula for this Euler-Poincaré characteristic in Dynkin type $A$ and affine Dynkin type $\tilde{A}$, see Theorem 8.1. In particular, we show that the EulerPoincaré characteristic is non-negative, see Corollary 8.2. We also study projective presentations in the module categories of finite dimensional algebras associated to the triangulations of the surfaces.

In [SZ, CZ, Z, MP] cluster expansions for cluster algebras of rank 2 are given. A related cluster expansion formula in the type $A$ case was given in $[\mathrm{Pr}$.

In [M] a cluster expansion for cluster algebras of finite classical type is given for clusters that lie in a bipartite seed. 
The paper is organized as follows. In section 2, we recall some definitions and results from the theory of cluster algebras. We state our formula for the Laurent polynomials in cluster algebras with principal coefficients in Theorem 3.2 in section 3. In that section, we also introduce complete $(T, \gamma)$-paths. Sections 4 and 5 are devoted to the proof of Theorem 3.2, section 4 deals with the simply connected case and section 5 with the general case. Section 5 also contains some necessary preparatory results on Galois coverings of quivers. In section 6, we present our formulas for $F$-polynomials and $g$-vectors, and prove Conjectures 5.4 and 5.5 of [FZ2]. A formula for the Laurent expansions in cluster algebras with arbitrary coefficients is given in section 7 In that section, we also prove the positivity conjecture for arbitrary coefficients of geometric type. In section 8.1. we study Euler-Poincaré characteristics of quiver Grassmannians and projective presentations in the module categories of finite dimensional algebras associated to triangulated surfaces in ABCP.

The author thanks Gregg Musiker and Hugh Thomas for several interesting discussions.

\section{Cluster algebras}

In this section, we recall some facts on cluster algebras. For further details, the reader is referred to FZ2, FST.

2.1. Definition. Following Fomin and Zelevinsky, let $(\mathbb{P}, \oplus, \cdot)$ be a semifield, i.e. an abelian group $(\mathbb{P}, \cdot)$ together with a binary operation $\oplus$ which is commutative, associative, and, with respect to the multiplication $\cdot$, distributive. Let $\mathbb{Z} \mathbb{P}$ be the group ring of $(\mathbb{P}, \cdot)$ and let $\mathcal{F}=\mathbb{Q P}\left(x_{1}, x_{2}, \ldots, x_{n}\right)$ be the field of rational functions in $n$ variables with coefficients in $\mathbb{Q P}$.

A seed $\Sigma$ is a triple $\Sigma=(\mathbf{x}, \mathbf{y}, B)$, where

- $\mathbf{x}=\left\{x_{1}, x_{2}, \ldots, x_{n}\right\}$ is a transcendence basis of $\mathcal{F}$ over $\mathbb{Q P}$,

- $\mathbf{y}=\left\{y_{1}, y_{2}, \ldots, y_{n}\right\}$ is an $n$-tuple of elements $y_{i} \in \mathbb{P}$, and

- $B=\left(b_{i j}\right)$ is a skew symmetrizable $n \times n$ integer matrix.

The set $\mathbf{x}$ is called a cluster and its elements are cluster variables. The set $\mathbf{y}$ is called coefficient tuple and $B$ is called exchange matrix.

Remark 2.1. In this paper we will study a special type of cluster algebras, those arising from surfaces, and for these cluster algebras the matrix $B$ is always skew symmetric.

Given a seed $(\mathbf{x}, \mathbf{y}, B)$ its mutation $\mu_{k}(\mathbf{x}, \mathbf{y}, B)$ in direction $k$ is a new seed $\left(\mathbf{x}^{\prime}, \mathbf{y}^{\prime}, B^{\prime}\right)$ defined as follows. Let $[x]_{+}=\max (x, 0)$.

- $B^{\prime}=\left(b_{i j}^{\prime}\right)$ with

$$
b_{i j}^{\prime}= \begin{cases}-b_{i j} & \text { if } i=k \text { or } j=k, \\ b_{i j}+\left[-b_{i k}\right]_{+} b_{k j}+b_{i k}\left[b_{k j}\right]_{+} & \text {otherwise, }\end{cases}
$$

- $\mathbf{y}^{\prime}=\left(y_{1}^{\prime}, y_{2}^{\prime}, \ldots, y_{n}^{\prime}\right)$ with

$$
y_{j}^{\prime}= \begin{cases}y_{k}^{-1} & \text { if } j=k, \\ y_{j} y_{k}^{\left[b_{k j}\right]_{+}}\left(y_{k} \oplus 1\right)^{-b_{k j} j} & \text { if } j \neq k,\end{cases}
$$


- $\mathbf{x}^{\prime}=\mathbf{x} \backslash\left\{x_{k}\right\} \cup\left\{x_{k}^{\prime}\right\}$ where

$$
x_{k}^{\prime}=\frac{y_{k} \prod x_{i}^{\left[b_{i k}\right]_{+}}+\prod x_{i}^{\left[-b_{i k}\right]_{+}}}{\left(y_{k} \oplus 1\right) x_{k}} .
$$

The formula (5) is called exchange relation. One can check that the mutations are involutions, that is, $\mu_{k} \mu_{k}(\mathbf{x}, \mathbf{y}, B)=(\mathbf{x}, \mathbf{y}, B)$.

Most of the time, we will be dealing with cluster algebras of geometric type, which means that $\mathbb{P}$ is a tropical semifield $\mathbb{P}=\operatorname{Trop}\left(u_{1}, \ldots, u_{\ell}\right)$, that is, $(\mathbb{P}, \cdot)$ is a free abelian group on the generators $u_{1}, u_{2}, \ldots, u_{\ell}$, and the addition $\oplus$ is given by the formula

$$
\prod_{j=1}^{\ell} u_{j}^{a_{j}} \oplus \prod_{j=1}^{\ell} u_{j}^{b_{j}}=\prod_{j=1}^{\ell} u_{j}^{\min \left(a_{j}, b_{j}\right)} .
$$

In cluster algebras of geometric type, it is convenient to replace the matrix $B$ by an $(n+\ell) \times n$ matrix $\tilde{B}=\left(b_{i j}\right)$ whose upper part is the $n \times n$ matrix $B$ and whose lower part is an $\ell \times n$ matrix that encodes the coefficient tuple via

$$
y_{k}=\prod_{i=1}^{\ell} u_{i}^{b_{(n+i) k}} .
$$

Then the mutation of the coefficient tuple in equation (44) is determined by the mutation of the matrix $\tilde{B}$ in equation (3) and the formula (6); and the exchange relation (5) becomes

$$
x_{k}^{\prime}=x_{k}^{-1}\left(\prod_{i=1}^{n} x_{i}^{\left[b_{i k}\right]_{+}} \prod_{i=1}^{\ell} u_{i}^{\left[b_{(b+i) k}\right]_{+}}+\prod_{i=1}^{n} x_{i}^{\left[-b_{i k}\right]_{+}} \prod_{i=1}^{\ell} u_{i}^{\left[-b_{(n+i) k}\right]_{+}}\right) .
$$

If the cluster algebra is of geometric type then the group ring $\mathbb{Z P}$ of $(\mathbb{P}, \cdot)$, is the ring of Laurent polynomials in the variables $u_{1}, u_{2}, \ldots, u_{\ell}$.

Two seeds $\Sigma_{1}, \Sigma_{2}$ are called mutation equivalent if there is a sequence of mutations $\mu=\mu_{i_{1}} \mu_{i_{2}} \cdots \mu_{i_{s}}$ such that $\mu \Sigma_{1}=\Sigma_{2}$. Thus starting from an initial seed $(\mathbf{x}, \mathbf{y}, B)$ one constructs the class of all seeds that are mutation equivalent to the initial one by successive mutations in all possible directions.

Define $\mathcal{X}$ to be the set of all cluster variables, that is, $\mathcal{X}$ is the union of all clusters $\mathbf{x}^{\prime}$ such that there exists a seed $\left(\mathbf{x}^{\prime}, \mathbf{y}^{\prime}, B^{\prime}\right)$ that is mutation equivalent to the initial seed. The cluster algebra $\mathcal{A}(\mathbf{x}, \mathbf{y}, B)$ is the $\mathbb{Z} \mathbb{P}$-subalgebra of the field $\mathcal{F}$ generated by the set of all cluster variables, thus

$$
\mathcal{A}(\mathbf{x}, \mathbf{y}, B)=\mathbb{Z} \mathbb{P}[\mathcal{X}] .
$$

Using the exchange relations, each cluster variable can be written as a rational function of the cluster variables in the initial seed. The following theorem, known as the Laurent phenomenon, states that these rational functions are actually Laurent polynomials.

Theorem 2.2. [FZ1, Theorem 3.1] Let $x \in \mathcal{X}$ be any cluster variable in the cluster algebra $\mathcal{A}(\mathbf{x}, \mathbf{y}, B)$. Then $x$ has an expansion in the initial cluster $\mathbf{x}=$ $\left\{x_{1}, x_{2}, \ldots, x_{n}\right\}$ as

$$
x=\frac{f\left(x_{1}, x_{2}, \ldots, x_{n}\right)}{x_{1}^{d_{1}} x_{2}^{d_{2}} \cdots x_{n}^{d_{n}}},
$$

where the right hand side is a reduced fraction and $f \in \mathbb{Z} \mathbb{P}\left[x_{1}, x_{2}, \ldots, x_{n}\right], d_{i} \geq 0$. 
It has been conjectured in [FZ1 that the polynomials $f$ have non-negative coefficients.

Conjecture 2.3 (Positivity Conjecture). Each coefficient of the polynomial $f$ in Theorem 2.2 is a non-negative integer linear combination of elements in $\mathbb{P}$.

2.2. Cluster algebras with principal coefficients. Fomin and Zelevinsky introduced in FZ2 a special type of coefficients, called principal coefficients. We recall some of their properties here.

A cluster algebra $\mathcal{A}=\mathcal{A}(\mathbf{x}, \mathbf{y}, B)$ is said to have principal coefficients if the coefficient semifield $\mathbb{P}$ is the tropical semifield $(\mathbb{P}, \oplus, \cdot)=\operatorname{Trop}\left(y_{1}, y_{2}, \ldots, y_{n}\right)$ with the initial coefficient tuple $\mathbf{y}=\left\{y_{1}, y_{2}, \ldots, y_{n}\right\}$ as set of generators.

In particular, $\mathcal{A}$ is of geometric type, so one can replace the $n \times n$ matrix $B$ by a $2 n \times n$ matrix $\tilde{B}$ whose upper part is the $n \times n$ matrix $B$ and whose lower part encodes the coefficient tuple $\mathbf{y}$ in the seed by formula (6). In this description, the cluster algebra $\mathcal{A}$ has principal coefficients if the lower part of the initial matrix $\tilde{B}$ is the $n \times n$ identity matrix.

Proposition 2.4. For cluster algebras with principal coefficients, the cluster expansions of Theorem 2.2 take the form

$$
x=\frac{f\left(x_{1}, x_{2}, \ldots, x_{n} ; y_{1}, y_{2}, \ldots, y_{n}\right)}{x_{1}^{d_{1}}, x_{2}^{d_{2}}, \ldots, x_{n}^{d_{n}}},
$$

where $f \in \mathbb{Z}\left[x_{1}, x_{2} \ldots, x_{n} ; y_{1}, y_{2}, \ldots, y_{n}\right]$.

Proof. [FZ2, Proposition 3.6]

Knowing the cluster expansions for a cluster algebra with principal coefficients allows one to compute the cluster expansions for the "same" cluster algebra with an arbitrary coefficient system. More precisely, let $\hat{A}=\mathcal{A}(\mathbf{x}, \hat{\mathbf{y}}, B)$ be a cluster algebra over $\mathbb{Z} \hat{\mathbb{P}}$ with initial coefficient tuple $\hat{\mathbf{y}}=\left(\hat{y}_{1}, \hat{y}_{2}, \ldots, \hat{y}_{n}\right)$, where $\hat{\mathbb{P}}$ is an arbitrary semifield. Denote by $\hat{\mathcal{F}}$ the field of rational functions in $n$ variables with coefficients in $\mathbb{Q} \hat{\mathbb{P}}$. Let $A=\mathcal{A}(\mathbf{x}, \mathbf{y}, B)$ be the cluster algebra with principal coefficients that has the same initial cluster and the same initial exchange matrix as $\hat{\mathcal{A}}$. Let $\hat{x}$ be any cluster variable in $\hat{\mathcal{A}}$ and let $\mu=\mu_{i_{1}} \mu_{i_{2}} \cdots \mu_{i_{s}}$ be a sequence of mutations such that $\hat{x} \in \mu(\mathbf{x})$ but $\hat{x} \notin \mu_{i_{2}} \cdots \mu_{i_{s}}(\mathbf{x})$. Using the same sequence of mutations in $\mathcal{A}$, let $x$ be the unique cluster variable in $\mathcal{A}$ such that $x \in \mu(\mathbf{x})$ but $x \notin \mu_{i_{2}} \cdots \mu_{i_{s}}(\mathbf{x})$, and let

$$
x=\frac{f\left(x_{1}, x_{2}, \ldots, x_{n} ; y_{1}, y_{2}, \ldots, y_{n}\right)}{x_{1}^{d_{1}} \ldots x_{n}^{d_{n}}},
$$

be the cluster expansion of $x$ in the initial seed $(\mathbf{x}, \mathbf{y}, B)$.

Theorem 2.5. [FZ2, Theorem 3.7] With the above notation, the cluster expansion of $\hat{x}$ in the initial cluster $(\mathbf{x}, \hat{\mathbf{y}}, B)$ in $\hat{\mathcal{A}}$ is

$$
\hat{x}=\frac{\left.f\right|_{\hat{\mathcal{F}}}\left(x_{1}, x_{2}, \ldots, x_{n} ; \hat{y}_{1}, \hat{y}_{2}, \ldots, \hat{y}_{n}\right)}{\left.x_{1}^{d_{1}} \ldots x_{n}^{d_{n}} f\right|_{\hat{\mathbb{P}}}\left(1,1, \ldots, 1 ; \hat{y}_{1}, \hat{y}_{2}, \ldots, \hat{y}_{n}\right)},
$$

where $\left.f\right|_{\hat{\mathcal{F}}}\left(x_{1}, x_{2}, \ldots, x_{n} ; \hat{y}_{1}, \hat{y}_{2}, \ldots, \hat{y}_{n}\right)$ is the polynomial $f$ evaluated in the field $(\hat{\mathcal{F}},+, \cdot)$ after substituting $\hat{y}_{i}$ for $y_{i}$, and $\left.f\right|_{\hat{\mathbb{P}}}\left(1,1, \ldots, 1 ; \hat{y}_{1}, \hat{y}_{2}, \ldots, \hat{y}_{n}\right)$ is the polynomial $f$ evaluated in the semifield $(\hat{\mathbb{P}}, \oplus, \cdot)$ after substituting 1 for $x_{i}$ and $\hat{y}_{i}$ for $y_{i}$, $i=1,2, \ldots, n$. 
Thus the cluster expansion of $\hat{x}$ is obtained from the expansion for $x$ by replacing $y_{i}$ by $\hat{y}_{i}$, and dividing by $\left.f\right|_{\hat{\mathbb{P}}}\left(1,1, \ldots, 1 ; \hat{y}_{1}, \hat{y}_{2}, \ldots, \hat{y}_{n}\right)$.

2.3. Cluster algebras arising from unpunctured surfaces. In this section, we recall the construction of [FST] in the case of surfaces without punctures.

Let $S$ be a connected oriented 2-dimensional Riemann surface with boundary and $M$ a non-empty set of marked points in the closure of $S$ with at least one marked point on each boundary component. The pair $(S, M)$ is called bordered surface with marked points. Marked points in the interior of $S$ are called punctures.

In this paper we will only consider surfaces $(S, M)$ such that all marked points lie on the boundary of $S$, and we will refer to $(S, M)$ simply by unpunctured surface. The orientation of the surface will play a crucial role.

We say that two curves in $S$ do not cross if they do not intersect each other except that endpoints may coincide.

Definition 1. An $\operatorname{arc} \gamma$ in $(S, M)$ is a curve in $S$ such that

(a) the endpoints are in $M$,

(b) $\gamma$ does not cross itself,

(c) the relative interior of $\gamma$ is disjoint from $M$ and from the boundary of $S$,

(d) $\gamma$ does not cut out a monogon or a digon.

Curves that connect two marked points and lie entirely on the boundary of $S$ without passing through a third marked point are called boundary arcs. Hence an arc is a curve between two marked points, which does not intersect itself nor the boundary except possibly at its endpoints and which is not homotopic to a point or a boundary arc.

Each arc is considered up to isotopy inside the class of such curves. Moreover, each arc is considered up to orientation, so if an arc has endpoints $a, b \in M$ then it can be represented by a curve that runs from $a$ to $b$, as well as by a curve that runs from $b$ to $a$.

For any two $\operatorname{arcs} \gamma, \gamma^{\prime}$ in $S$, let $e\left(\gamma, \gamma^{\prime}\right)$ be the minimal number of crossings of $\gamma$ and $\gamma^{\prime}$, that is, $e\left(\gamma, \gamma^{\prime}\right)$ is the minimum of the numbers of crossings of arcs $\alpha$ and $\alpha^{\prime}$, where $\alpha$ is isotopic to $\gamma$ and $\alpha^{\prime}$ is isotopic to $\gamma^{\prime}$. Two arcs $\gamma, \gamma^{\prime}$ are called compatible if $e\left(\gamma, \gamma^{\prime}\right)=0$. A triangulation is a maximal collection of compatible arcs together with all boundary arcs. The arcs of a triangulation cut the surface into triangles. Since $(S, M)$ is an unpunctured surface, the three sides of each triangle are distinct (in contrast to the case of surfaces with punctures). Any triangulation has $n+m$ elements, $n$ of which are arcs in $S$, and the remaining $m$ elements are boundary arcs. Note that the number of boundary arcs is equal to the number of marked points.

Proposition 2.6. The number $n$ of arcs in any triangulation is given by the formula $n=6 g+3 b+m-6$, where $g$ is the genus of $S, b$ is the number of boundary components and $m=|M|$ is the number of marked points. The number $n$ is called the rank of $(S, M)$.

Proof. [FST, 2.10]

Corollary 2.7. The number of triangles in any triangulation is equal to

$$
n-2(g-1)-b \text {. }
$$




\begin{tabular}{c|c|c||l}
$\mathrm{b}$ & $\mathrm{g}$ & $\mathrm{m}$ & surface \\
\hline 1 & 0 & $\mathrm{n}+3$ & polygon \\
1 & 1 & $\mathrm{n}-3$ & torus with disk removed \\
1 & 2 & $\mathrm{n}-9$ & genus 2 surface with disk removed \\
\hline 2 & 0 & $\mathrm{n}$ & annulus \\
2 & 1 & $\mathrm{n}-6$ & torus with 2 disks removed \\
2 & 2 & $\mathrm{n}-12$ & genus 2 surface with 2 disks removed \\
\hline 3 & 0 & $\mathrm{n}-3$ & pair of pants
\end{tabular}

TABLE 1. Examples of unpunctured surfaces

Proof. Consider the Riemann surface without boundary obtained from $S$ by gluing a disk into each boundary component. Computing the Euler-Poincaré characteristic of this surface using the genus on the one hand and using the simplicial complex given by the triangulation $T$ on the other hand leads to the equation

$$
2-2 g=\text { number of triangles }+b-(n+m)+m,
$$

and the statement follows.

Note that $b>0$ since the set $M$ is not empty. Table 1 gives some examples of unpunctured surfaces.

Following [FST, we associate a cluster algebra to the unpunctured surface $(S, M)$ as follows. Choose any triangulation $T$, let $\tau_{1}, \tau_{2}, \ldots, \tau_{n}$ be the $n$ interior $\operatorname{arcs}$ of $T$ and denote the $m$ boundary arcs of the surface by $\tau_{n+1}, \tau_{n+2}, \ldots, \tau_{n+m}$. For any triangle $\Delta$ in $T$ define a matrix $B^{\Delta}=\left(b_{i j}^{\Delta}\right)_{1 \leq i \leq n, 1 \leq j \leq n}$ by

$$
b_{i j}^{\Delta}= \begin{cases}1 & \text { if } \tau_{i} \text { and } \tau_{j} \text { are sides of } \Delta \text { with } \tau_{j} \text { following } \tau_{i} \text { in the } \\ -1 & \text { if } \tau_{i} \text { and } \tau_{j} \text { are sides of } \Delta \text { with } \tau_{j} \text { following } \tau_{i} \text { in the } \\ & \text { clockwise order; } \\ 0 \quad \text { otherwise }\end{cases}
$$

Then define the matrix $B_{T}=\left(b_{i j}\right)_{1 \leq i \leq n, 1 \leq j \leq n}$ by $b_{i j}=\sum_{\Delta} b_{i j}^{\Delta}$, where the sum is taken over all triangles in $T$. Note that the boundary arcs of the triangulation are ignored in the definition of $B_{T}$. Let $\tilde{B}_{T}=\left(b_{i j}\right)_{1 \leq i \leq 2 n, 1 \leq j \leq n}$ be the $2 n \times n$ matrix whose upper $n \times n$ part is $B_{T}$ and whose lower $n \times n$ part is the identity matrix. The matrix $B_{T}$ is skew-symmetric and each of its entries $b_{i j}$ is either $0,1,-1,2$, or -2 , since every arc $\tau$ can be in at most two triangles. An example where $b_{i j}=2$ is given in Figure 1 .

Let $\mathcal{A}\left(\mathbf{x}_{T}, \mathbf{y}_{T}, B_{T}\right)$ be the cluster algebra with principal coefficients in the triangulation $T$, that is, $\mathcal{A}\left(\mathbf{x}_{T}, \mathbf{y}_{T}, B_{T}\right)$ is given by the seed $\left(\mathbf{x}_{T}, \mathbf{y}_{T}, B_{T}\right)$ where $\mathbf{x}_{T}=\left\{x_{\tau_{1}}, x_{\tau_{2}}, \ldots, x_{\tau_{n}}\right\}$ is the cluster associated to the triangulation $T$, and the initial coefficient vector $\mathbf{y}_{T}=\left(y_{1}, y_{2}, \ldots, y_{n}\right)$ is the vector of generators of $\mathbb{P}=\operatorname{Trop}\left(y_{1}, y_{2}, \ldots, y_{n}\right)$.

For the boundary arcs we define $x_{\tau_{k}}=1, k=n+1, n+2, \ldots, n+m$.

For each $k=1,2, \ldots, n$, there is a unique quadrilateral in $T \backslash\left\{\tau_{k}\right\}$ in which $\tau_{k}$ is one of the diagonals. Let $\tau_{k}^{\prime}$ denote the other diagonal in that quadrilateral. Define the flip $\mu_{k} T$ to be the triangulation $T \backslash\left\{\tau_{k}\right\} \cup\left\{\tau_{k}^{\prime}\right\}$. The mutation $\mu_{k}$ of the seed $\Sigma_{T}$ in the cluster algebra $\mathcal{A}$ corresponds to the flip $\mu_{k}$ of the triangulation 


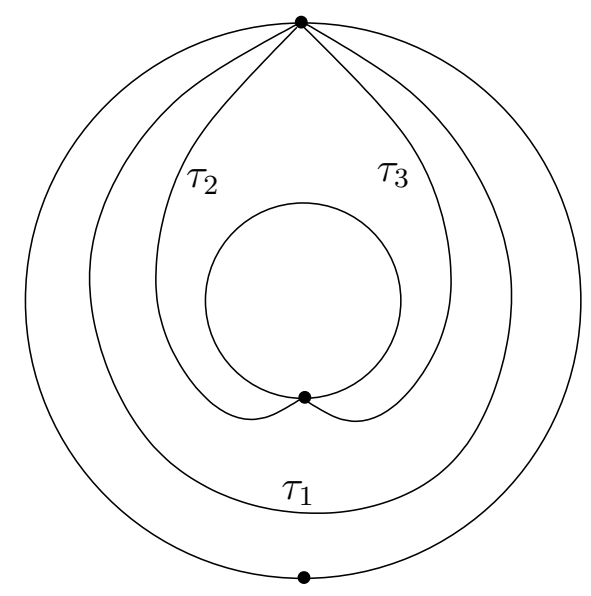

FIgURE 1. A triangulation with $b_{23}=2$

$T$ in the following sense. The matrix $\mu_{k}\left(B_{T}\right)$ is the matrix corresponding to the triangulation $\mu_{k} T$, the cluster $\mu_{k}\left(\mathbf{x}_{T}\right)$ is $\mathbf{x}_{T} \backslash\left\{x_{\tau_{k}}\right\} \cup\left\{x_{\tau_{k}^{\prime}}\right\}$, and the corresponding exchange relation is given by

$$
x_{\tau_{k}} x_{\tau_{k}^{\prime}}=x_{\rho_{1}} x_{\rho_{2}} y^{+}+x_{\sigma_{1}} x_{\sigma_{2}} y^{-},
$$

where $y^{+}, y^{-}$are some coefficients, and $\rho_{1}, \sigma_{1}, \rho_{2}, \sigma_{2}$ are the sides of the quadrilateral in which $\tau_{k}$ and $\tau_{k}^{\prime}$ are the diagonals, such that $\rho_{1}, \rho_{2}$ are opposite sides and $\sigma_{1}, \sigma_{2}$ are opposite sides too.

2.4. The quiver of a triangulation. Since the cluster algebra $\mathcal{A}=\mathcal{A}\left(\mathbf{x}_{T}, \mathbf{y}_{T}, B_{T}\right)$ defined in section 2.3 has principal coefficients, we can consider the $(2 n \times n)$ matrix $\tilde{B}_{T}$ whose upper part is $B_{T}$ and whose lower part is the $n \times n$ identity matrix. If $\mu$ is a sequence of mutations, then the upper part of $\mu \tilde{B}_{T}$ corresponds to the triangulation $\mu T$, but the triangulation does not give any information about the lower $n \times n$ part of the matrix $\widetilde{B}_{T}$.

In order to keep track of the whole matrix and, thus, the coefficient tuple, we find it convenient to use quivers.

Let $T$ be the triangulation that will serve as initial seed for the cluster algebra with principal coefficients. Define a quiver $Q_{T}$ as follows. The vertices of $Q_{T}$ are labeled by integers $1,2, \ldots, 2 n$, where the first $n$ vertices correspond to the interior $\operatorname{arcs} \tau_{1}, \ldots, \tau_{n}$ of $T$, and the second $n$ vertices correspond to the initial coefficient tuple $y_{1}, \ldots, y_{n}$. The arrows of $Q_{T}$ are given by the matrix $\tilde{B}_{T}=\left(\tilde{b}_{i j}\right)$, that is, for each pair $i>j$ of vertices, the quiver $Q_{T}$ has $\tilde{b}_{i j}$ arrows from $i$ to $j$; where we use the convention that, if $\tilde{b}_{i j}<0$ then we have $-\tilde{b}_{i j}$ arrows from $j$ to $i$.

In $\mathrm{ABCP}$, generalizing a construction of [CCS], the authors define relations for the full subquiver of $Q_{T}$ whose set of vertices is $\{1,2, \ldots, n\}$ and show that the corresponding bound quiver algebra is a gentle algebra. In the case where the surface is a polygon, respectively an annulus, these algebras are precisely the cluster-tilted algebras of type $A_{n}$, respectively $\tilde{A}_{n}$. In section 8.2 , we will study projective presentations of certain indecomposable modules over these algebras.

Independently, these relations have also been defined in $[\mathrm{LF}]$ using quivers with potentials introduced in $\mathrm{DWZ}$. In $[\mathrm{LF}$, the author considers the more general 
situation where the surface is allowed to have punctures. In the punctured case, the resulting bound quiver algebras are no longer gentle.

\section{Cluster expansions With PRincipal COEFficients}

In this section we state our formula for the cluster expansions with principal coefficients in Theorem 3.2

Let $T=\left\{\tau_{1}, \ldots, \tau_{n}, \tau_{n+1}, \ldots, \tau_{n+m}\right\}$ be a triangulation of the unpunctured surface $(S, M)$, where $\tau_{1}, \ldots, \tau_{n}$ are arcs and $\tau_{n+1}, \ldots, \tau_{n+m}$ are boundary arcs $(m=|M|)$. Let $B_{T}$ be the corresponding $2 n \times n$ matrix with lower half equal to the $n \times n$ identity matrix, and let $\mathcal{A}=\mathcal{A}\left(\mathbf{x}_{T}, \mathbf{y}_{T}, B_{T}\right)$ be the cluster algebra with principal coefficients and initial seed $\left(\mathbf{x}_{T}, \mathbf{y}_{T}, B_{T}\right)$, where $\mathbf{x}_{T}=\left\{x_{\tau_{1}}, \ldots, x_{\tau_{n}}\right\}$ is the initial cluster and $\mathbf{y}_{T}=\left\{y_{\tau_{1}}, \ldots, y_{\tau_{n}}\right\}$ is the initial coefficient vector. We will often write $x_{i}$ and $y_{i}$ instead of $x_{\tau_{i}}$ and $y_{\tau_{i}}$ respectively. By [FST], the cluster variables in $\mathcal{A}$ correspond to the arcs in $(S, M)$.

Let $\gamma$ be any arc in $(S, M)$ that crosses $T$ exactly $d$ times. We fix an orientation for $\gamma$ and we denote its starting point by $s$ and its endpoint by $t$, with $s, t \in M$, see Figure 2, Let $s=p_{0}, p_{1}, \ldots, p_{d}, p_{d+1}=t$ be the intersection points of $\gamma$ and $T$ in order of occurrence on $\gamma$, hence $p_{0}, p_{d+1} \in M$ and each $p_{i}$ with $1 \leq i \leq d$ lies in the interior of $S$. Let $i_{1}, i_{2}, \ldots, i_{d}$ be such that $p_{k}$ lies on the arc $\tau_{i_{k}} \in T$, for $k=1,2, \ldots, d$. Note that $i_{k}$ may be equal to $i_{j}$ even if $k \neq j$.

For $k=0,1, \ldots, d$, let $\gamma_{k}$ denote the segment of the path $\gamma$ from the point $p_{k}$ to the point $p_{k+1}$. Each $\gamma_{k}$ lies in exactly one triangle $\Delta_{k}$ in $T$. If $1 \leq k \leq d-1$, the triangle $\Delta_{k}$ is formed by the $\operatorname{arcs} \tau_{i_{k}}, \tau_{i_{k+1}}$ and a third arc that we denote by $\tau_{\left[\gamma_{k}\right]}$. In the triangle $\Delta_{0}, \tau_{i_{1}}$ is one of the sides. Denote the side of $\Delta_{0}$ that lies clockwise of $\tau_{i_{1}}$ by $\tau_{\left[\gamma_{0}\right]}$ and the other side by $\tau_{\left[\gamma_{-1}\right]}$. Similarly, $\tau_{i_{d}}$ is one of the sides of $\Delta_{d}$. Denote the side that lies clockwise of $\tau_{i_{d}}$ by $\tau_{\left[\gamma_{d}\right]}$ and the other side by $\tau_{\left[\gamma_{d+1}\right]}$.

3.1. Complete $(T, \gamma)$-paths. A $T$-path is a path $\alpha$ in $S$ on the triangulation $T$, that is, there exist arcs $\alpha_{1}, \alpha_{2}, \ldots, \alpha_{\ell(\alpha)} \in T$ such that $\alpha$ is the concatenation of paths $\alpha=\alpha_{1} \alpha_{2} \cdots \alpha_{\ell(\alpha)}$. We will write $\alpha=\left(\alpha_{1}, \alpha_{2}, \ldots, \alpha_{\ell(\alpha)}\right)$. Recall that arcs in $(S, M)$ are not oriented. Now a $T$-path induces an orientation on each of its $\operatorname{arcs} \alpha_{i}$. Note that a $T$-path $\alpha$ may not be uniquely determined by the sequence $\left(\alpha_{1}, \alpha_{2}, \ldots, \alpha_{\ell(\alpha)}\right)$ since arcs may be loops and, thus, picking a starting point and an endpoint does not determine the orientation. However, the sequences $\left(\alpha_{1}, \alpha_{2}, \ldots, \alpha_{\ell(\alpha)}\right)$ determine the complete $(T, \gamma)$-paths that we are going to define now.

Definition 2. MS A T-path $\alpha=\left(\alpha_{1}, \alpha_{2}, \ldots, \alpha_{\ell(\alpha)}\right)$ is called a complete $(T, \gamma)$ path if the following axioms hold:

(T1) The even arcs are precisely the arcs crossed by $\gamma$ in order, that is, $\alpha_{2 k}=\tau_{i_{k}}$.

(T2) For all $k=0,1,2, \ldots, d$, the segment $\gamma_{k}$ is homotopic to the segment of the path $\alpha$ starting at the point $p_{k}$ following $\alpha_{2 k}, \alpha_{2 k+1}$ and $\alpha_{2 k+2}$ until the point $p_{k+1}$.

Remark 3.1. - Every complete $(T, \gamma)$-path starts and ends at the same point as $\gamma$, because of (T2).

- Every complete $(T, \gamma)$-path has length $2 d+1$.

- For all arcs $\tau$ in the triangulation $T$, the number of times that $\tau$ occurs as $\alpha_{2 k}$ is exactly the number of crossings between $\gamma$ and $\tau$. 


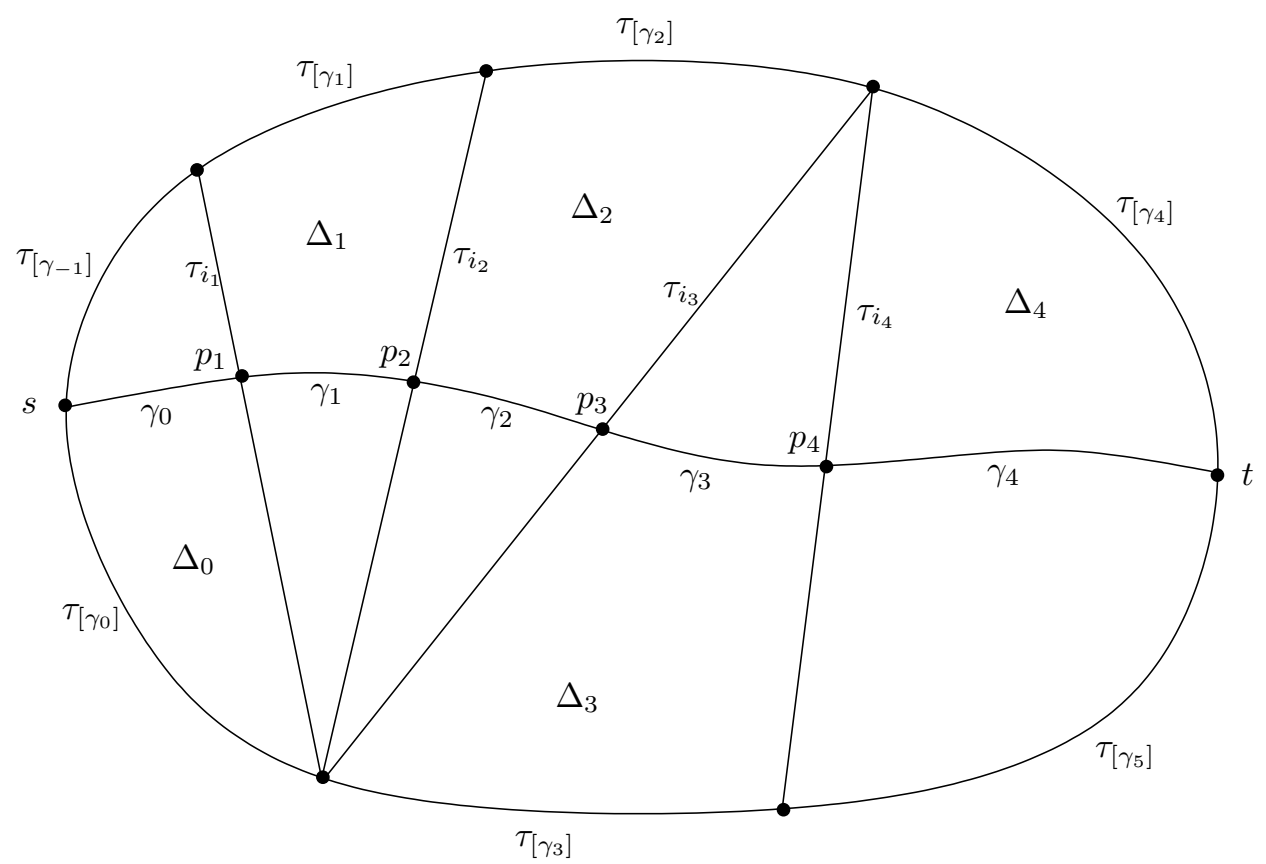

Figure 2. Relative position of $\gamma$ and $T$, an example with $d=4$

- In contrast to the ordinary $(T, \gamma)$-paths introduced in $\underline{\mathrm{ST}}$, complete $(T, \gamma)$ paths allow backtracking.

3.2. Orientation. By Corollary 2.7 the triangulation $T$ cuts the surface $S$ into $(n-2(g-1)-b)$ triangles. The orientation of the surface $S$ induces an orientation on each of these triangles in such a way that, whenever two triangles $\Delta, \Delta^{\prime}$ share an edge $\tau$, then the orientation of $\tau$ in $\Delta$ is opposite to the orientation of $\tau$ in $\Delta^{\prime}$, see Figure 3. There are precisely two such orientations, we assume without loss of generality that we have the "clockwise orientation", that is, in each triangle $\Delta$, going around the boundary of $\Delta$ according to the orientation of $\Delta$ is clockwise when looking at it from outside the surface.

Let $\alpha$ be a complete $(T, \gamma)$-path. Then $\alpha_{2 k}=\tau_{i_{k}}$ is a common edge of the two triangles $\Delta_{k-1}$ and $\Delta_{k}$. We say that $\alpha_{2 k}$ is $\gamma$-oriented if the orientation of $\alpha_{2 k}$ in the path $\alpha$ is the same as the orientation of $\tau_{i_{k}}$ in the triangle $\Delta_{k}$, see Figure 4

3.3. Expansion Formula. We are ready to state the main result of this section. We keep the setup of the previous sections. Define

$$
x(\alpha)=\frac{\prod_{k \text { odd }} x_{\alpha_{k}}}{x_{i_{1}} x_{i_{2}} \ldots x_{i_{d}}} \quad \text { and } \quad y(\alpha)=\prod_{k: \alpha_{2 k}} \prod_{\text {is } \gamma \text {-oriented }} y_{i_{k}}
$$

Thus $x(\alpha)$ does not depend on the orientation, but $y(\alpha)$ does. Recall that $x_{\alpha_{k}}=1$ if $\alpha_{k}$ is a boundary arc.

The following theorem gives a cluster expansion formula of an arbitrary cluster variable $x_{\gamma}$ in the initial cluster with principal coefficients. 


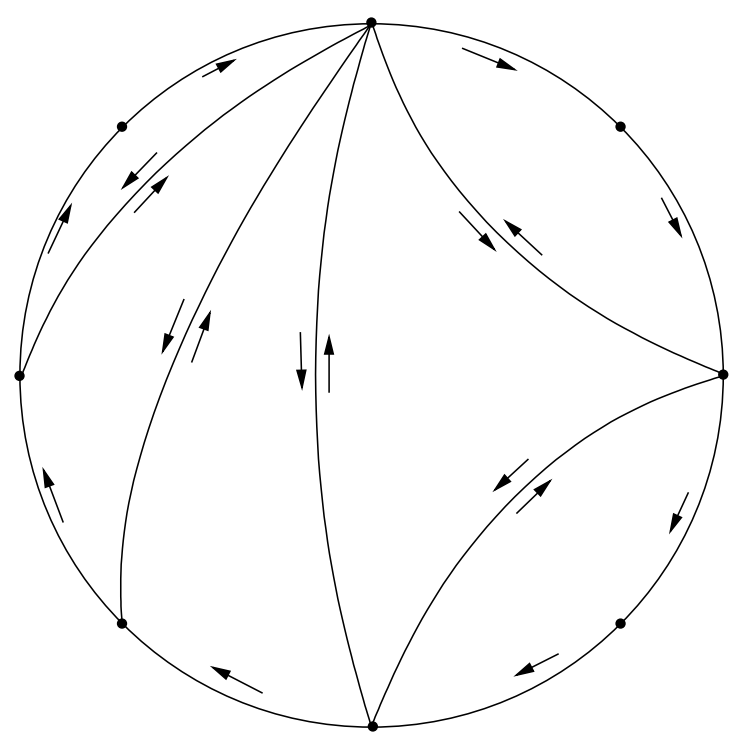

Figure 3. A triangulation of the disk with 8 marked points with clockwise orientation
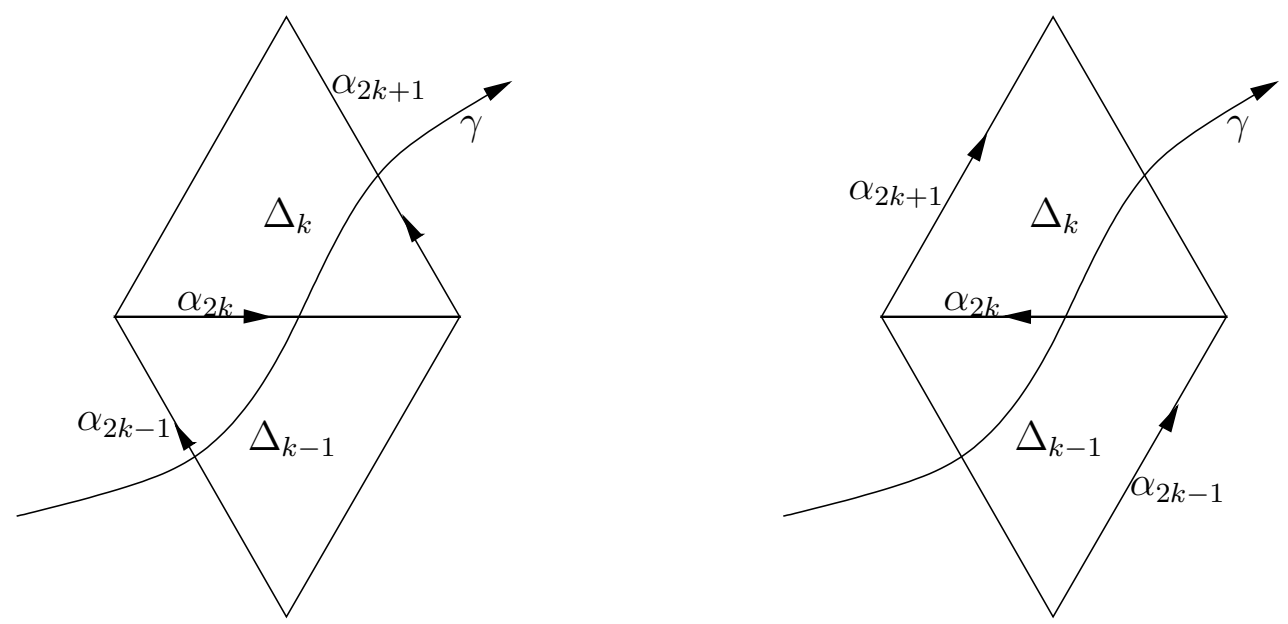

Figure 4. Two examples of the $(T, \gamma)$-path segment $\left(\alpha_{2 k-1}, \alpha_{2 k}, \alpha_{2 k+1}\right)$. On the left, $\alpha_{2 k}$ is not $\gamma$-oriented and on the right, $\alpha_{2 k}$ is $\gamma$-oriented.

Theorem 3.2. Let $x_{\gamma}$ be any cluster variable in the cluster algebra $\mathcal{A}$. Then its expansion in the initial seed $\left(\mathbf{x}_{T}, \mathbf{y}_{T}, B_{T}\right)$ is given by

$$
x_{\gamma}=\sum_{\alpha} x(\alpha) y(\alpha)
$$

where the sum is over all complete $(T, \gamma)$-paths $\alpha$ in $(S, M)$.

Remark 3.3. The formula in Theorem 3.2 does not depend on our choice of orientation on the arc $\gamma$. Indeed, considering the opposite orientation $\gamma^{o p}$, there is a 
bijection between the set of complete $(T, \gamma)$-paths and complete $\left(T, \gamma^{o p}\right)$-paths, sending a path $\alpha=\left(\alpha_{1}, \alpha_{2}, \ldots, \alpha_{2 d+1}\right)$ to the opposite path $\alpha^{\text {op }}=\left(\alpha_{2 d+1}, \ldots, \alpha_{2}, \alpha_{1}\right)$; moreover $x\left(\alpha^{o p}\right)=x(\alpha)$, and $y\left(\alpha^{o p}\right)=y(\alpha)$.

The proof of Theorem 3.2 will be given in section 4 for simply connected surfaces and in section 5 for arbitrary surfaces. To illustrate the statement, we give two examples here.

Example 3.4. The case $A_{n}$ : The cluster algebra $\mathcal{A}$ is of type $A_{n}$ if $(S, M)$ is an $(n+3)$-gon. Our example illustrates the case $n=5$. The following figure shows a triangulation $T=\left\{\tau_{1}, \ldots, \tau_{13}\right\}$ and a (dotted) arc $\gamma$. Next to it is the list of complete $(T, \gamma)$-paths.

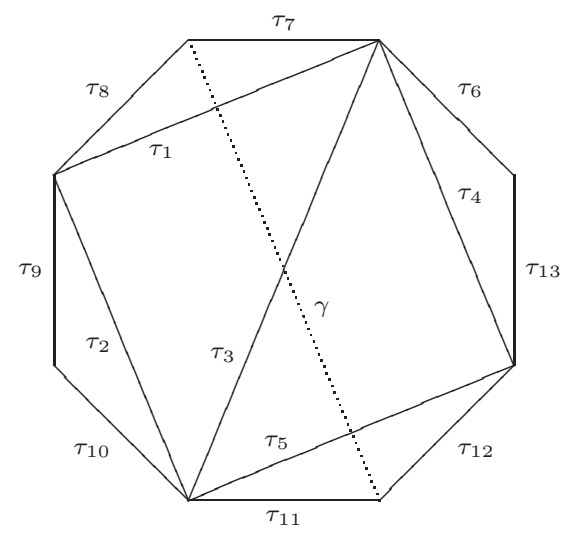

$$
\begin{aligned}
& \left(\tau_{8}, \tau_{1}, \tau_{3}, \tau_{3}, \tau_{3}, \tau_{5}, \tau_{12}\right) \\
& \left(\tau_{8}, \tau_{1}, \tau_{3}, \tau_{3}, \tau_{4}, \tau_{5}, \tau_{11}\right) \\
& \left(\tau_{7}, \tau_{1}, \tau_{2}, \tau_{3}, \tau_{3}, \tau_{5}, \tau_{12}\right) \\
& \left(\tau_{7}, \tau_{1}, \tau_{2}, \tau_{3}, \tau_{4}, \tau_{5}, \tau_{11}\right) \\
& \left(\tau_{7}, \tau_{1}, \tau_{1}, \tau_{3}, \tau_{5}, \tau_{5}, \tau_{11}\right)
\end{aligned}
$$

Theorem 3.2 thus implies that

$$
\begin{aligned}
x_{\gamma}= & \left(x_{1} x_{3} x_{5}\right)^{-1} \quad\left(x_{3}^{2} y_{1} y_{3} y_{5}\right. \\
& +x_{3} x_{4} y_{1} y_{3} \\
& +x_{2} x_{3} y_{3} y_{5} \\
& +x_{2} x_{4} y_{3} \\
& \left.+x_{1} x_{5}\right)
\end{aligned}
$$

Example 3.5. The case $\tilde{A}_{n-1}$ : The cluster algebra $\mathcal{A}$ is of type $\tilde{A}_{n-1}$ if $(S, M)$ is an annulus. Our example illustrates the case $n=4$. Figure 5 shows a triangulation $T=\left\{\tau_{1}, \tau_{2}, \ldots, \tau_{8}\right\}$ and $a$ (dotted) arc $\gamma$. The complete list of $(T, \gamma)$-paths is as 
follows:

$$
\begin{aligned}
& \left(\tau_{4}, \tau_{1}, \tau_{2}, \tau_{2}, \tau_{2}, \tau_{3}, \tau_{4}, \tau_{4}, \tau_{4}, \tau_{1}, \tau_{2}\right) \\
& \left(\tau_{4}, \tau_{1}, \tau_{2}, \tau_{2}, \tau_{2}, \tau_{3}, \tau_{4}, \tau_{4}, \tau_{5}, \tau_{1}, \tau_{8}\right) \\
& \left(\tau_{4}, \tau_{1}, \tau_{2}, \tau_{2}, \tau_{6}, \tau_{3}, \tau_{7}, \tau_{4}, \tau_{4}, \tau_{1}, \tau_{2}\right) \\
& \left(\tau_{4}, \tau_{1}, \tau_{2}, \tau_{2}, \tau_{6}, \tau_{3}, \tau_{7}, \tau_{4}, \tau_{5}, \tau_{1}, \tau_{8}\right) \\
& \left(\tau_{4}, \tau_{1}, \tau_{2}, \tau_{2}, \tau_{6}, \tau_{3}, \tau_{3}, \tau_{4}, \tau_{1}, \tau_{1}, \tau_{8}\right) \\
& \\
& \\
& \left(\tau_{5}, \tau_{1}, \tau_{8}, \tau_{2}, \tau_{2}, \tau_{3}, \tau_{4}, \tau_{4}, \tau_{4}, \tau_{1}, \tau_{2}\right) \\
& \left(\tau_{5}, \tau_{1}, \tau_{8}, \tau_{2}, \tau_{2}, \tau_{3}, \tau_{4}, \tau_{4}, \tau_{5}, \tau_{1}, \tau_{8}\right) \\
& \left(\tau_{5}, \tau_{1}, \tau_{8}, \tau_{2}, \tau_{6}, \tau_{3}, \tau_{7}, \tau_{4}, \tau_{4}, \tau_{1}, \tau_{2}\right) \\
& \left(\tau_{5}, \tau_{1}, \tau_{8}, \tau_{2}, \tau_{6}, \tau_{3}, \tau_{7}, \tau_{4}, \tau_{5}, \tau_{1}, \tau_{8}\right) \\
& \left(\tau_{5}, \tau_{1}, \tau_{8}, \tau_{2}, \tau_{6}, \tau_{3}, \tau_{3}, \tau_{4}, \tau_{1}, \tau_{1}, \tau_{8}\right) \\
& \left(\tau_{5}, \tau_{1}, \tau_{1}, \tau_{2}, \tau_{3}, \tau_{3}, \tau_{7}, \tau_{4}, \tau_{4}, \tau_{1}, \tau_{2}\right) \\
& \left(\tau_{5}, \tau_{1}, \tau_{1}, \tau_{2}, \tau_{3}, \tau_{3}, \tau_{7}, \tau_{4}, \tau_{5}, \tau_{1}, \tau_{8}\right) \\
& \left(\tau_{5}, \tau_{1}, \tau_{1}, \tau_{2}, \tau_{3}, \tau_{3}, \tau_{3}, \tau_{4}, \tau_{1}, \tau_{1}, \tau_{8}\right)
\end{aligned}
$$

Hence Theorem 3.2 implies

$$
\begin{aligned}
x_{\gamma}= & \left(x_{1} x_{2} x_{3} x_{4} x_{1}\right)^{-1}\left(x_{4} x_{2} x_{2} x_{4} x_{4} x_{2} y_{1} y_{2} y_{3} y_{4} y_{1}\right. \\
& +x_{4} x_{2} x_{2} x_{4} y_{1} y_{2} y_{3} y_{4} \\
& +x_{4} x_{2} x_{4} x_{2} y_{1} y_{2} y_{4} y_{1} \\
& +x_{4} x_{2} y_{1} y_{2} y_{4} \\
& +x_{4} x_{2} x_{3} x_{1} y_{1} y_{2} \\
& +x_{2} x_{4} x_{4} x_{2} y_{2} y_{3} y_{4} y_{1} \\
& +x_{2} x_{4} y_{2} y_{3} y_{4} \\
& +x_{4} x_{2} y_{2} y_{4} y_{1} \\
& +y_{2} y_{4} \\
& +x_{3} x_{1} y_{2} \\
& +x_{1} x_{3} x_{4} x_{2} y_{4} y_{1} \\
& +x_{1} x_{3} y_{4} \\
& \left.x_{1} x_{3} x_{3} x_{1}\right)
\end{aligned}
$$

Note that the term $x_{2}^{2} x_{4}^{2} y_{1} y_{2} y_{3} y_{4}$ appears with multiplicity two.

3.4. Positivity. The following positivity conjecture of [FZ1] is a direct consequence of Theorem 3.2 .

Corollary 3.6. Let $(S, M)$ be an unpunctured surface and let $\mathcal{A}=\mathcal{A}(\mathbf{x}, \mathbf{y}, B)$ be the cluster algebra with principal coefficients in the seed $(\mathbf{x}, \mathbf{y}, B)$ associated to some triangulation of $(S, M)$. Let $u$ be any cluster variable and let

$$
x=\frac{f\left(x_{1}, \ldots, x_{n}, y_{1}, \ldots, y_{n}\right)}{x_{1}^{d_{1}} \ldots x_{n}^{d_{n}}}
$$

be the expansion of $u$ in the cluster $\mathbf{x}\left\{x_{1}, \ldots, x_{n}\right\}$, where $f$ is a polynomial which is not divisible by any of the $x_{1}, \ldots, x_{n}$. Then the coefficients of $f$ are non-negative integers.

Proof. This is a direct consequence of Theorem 3.2 


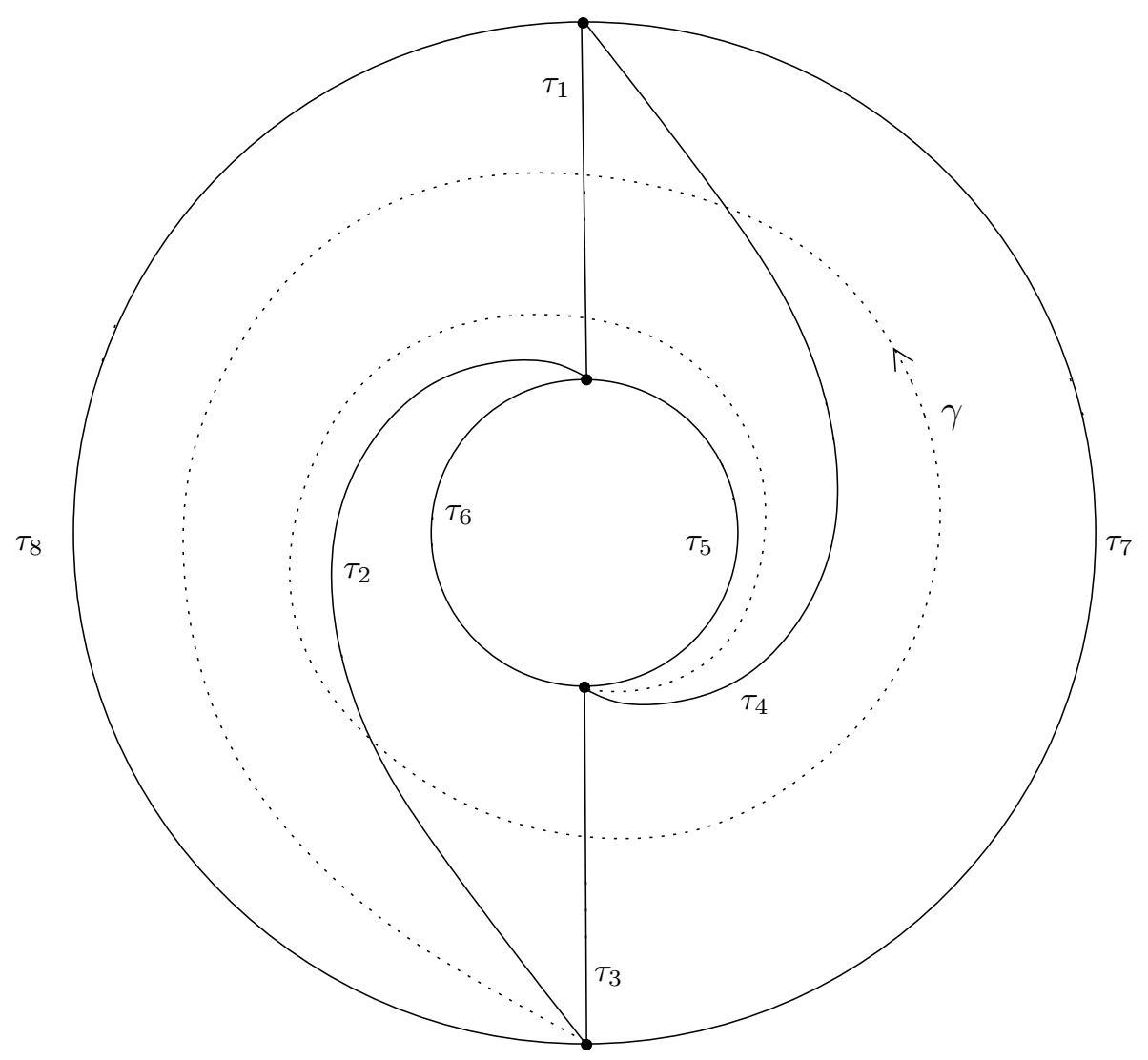

Figure 5. The case $\tilde{A}_{n-1}$

Remark 3.7. We will also prove this conjecture for arbitrary coefficients of geometric type in Theorem 7.1 .

\section{Proof of Theorem 3.2 for Simply Connected surfaces}

In this section, we prove Theorem 3.2 in the case where the surface $S$ is simply connected.

Recall that $T=\left\{\tau_{1}, \ldots, \tau_{n}, \tau_{n+1}, \ldots, \tau_{n+m}\right\}$ is a triangulation, $\gamma$ is an arc on which we fixed an orientation such that $\gamma$ is going from $s$ to $t, \gamma$ crosses $T$ exactly $d$ times, $s=p_{0}, p_{1}, \ldots, p_{d}, p_{d+1}=t$ are the intersection points of $\gamma$ and $T$ in order of occurrence on $\gamma$, and $i_{1}, i_{2}, \ldots, i_{d}$ are such that $p_{k}$ lies on the $\operatorname{arc} \tau_{i_{k}} \in T$, for $k=1,2, \ldots, d$.

Also recall that $\gamma_{k}$ denotes the segment of the path $\gamma$ from the point $p_{k}$ to the point $p_{k+1}$. Each $\gamma_{k}$ lies in exactly one triangle $\Delta_{k}$ in $T$. If $1 \leq k \leq d-1$, the triangle $\Delta_{k}$ is formed by the arcs $\tau_{i_{k}}, \tau_{i_{k+1}}$ and a third arc that we denote by $\tau_{\left[\gamma_{k}\right]}$.

Lemma 4.1. If $S$ is simply connected then

(a) Any two arcs in $S$ cross at most once.

(b) Two paths are homotopic if they have the same startpoint and the same endpoint. In particular, any arc is uniquely determined by its endpoints. 


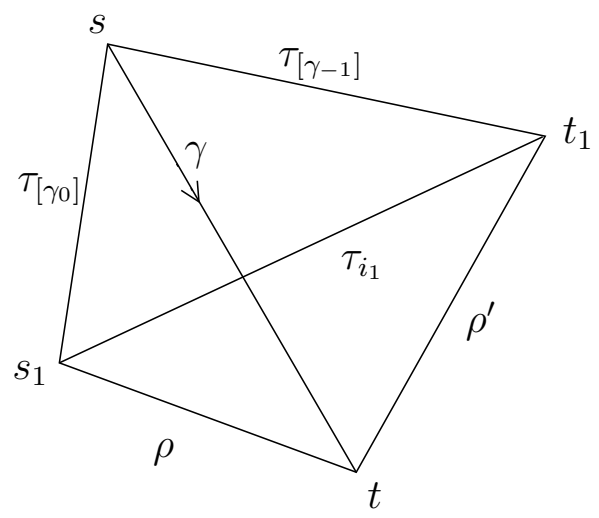

Figure 6. Proof of the simply connected case.

(c) $i_{k}=i_{j}$ if and only if $k=j$.

(d) $\Delta_{k}=\Delta_{j}$ if and only if $k=j$.

In the triangle $\Delta_{0}, \tau_{i_{1}}$ is one of the sides. Denote the side of $\Delta_{0}$ that lies clockwise of $\tau_{i_{1}}$ by $\tau_{\left[\gamma_{0}\right]}$ and the other side by $\tau_{\left[\gamma_{-1}\right]}$, see Figure 6 Similarly, $\tau_{i_{d}}$ is one of the sides of $\Delta_{d}$. Denote the side that lies clockwise of $\tau_{i_{d}}$ by $\tau_{\left[\gamma_{d}\right]}$ and the other side by $\tau_{\left[\gamma_{d+1}\right]}$. Note that $\tau_{\left[\gamma_{-1}\right]}$ and $\tau_{\left[\gamma_{0}\right]}$ may be boundary arcs. Let $s_{1}$ be the common endpoint of $\tau_{i_{1}}$ and $\tau_{\left[\gamma_{0}\right]}$ and let $t_{1}$ be the common endpoint of $\tau_{i_{1}}$ and $\tau_{\left[\gamma_{-1}\right]}$. Since $S$ is simply connected, there is a unique $\operatorname{arc} \rho$ from $s_{1}$ to $t$ and a unique $\operatorname{arc} \rho^{\prime}$ from $t_{1}$ to $t$, and, moreover, the $\operatorname{arcs} \tau_{\left[\gamma_{-1}\right]}, \tau_{\left[\gamma_{0}\right]}, \rho$, and $\rho^{\prime}$ form a quadrilateral in which $\gamma$ and $\tau_{i_{1}}$ are the diagonals, see Figure 6 .

With this notation, we have the following Lemma.

Lemma 4.2. If $S$ is simply connected, then any arc $\tau \in T$ that crosses $\rho$ (respectively $\rho^{\prime}$ ) also crosses $\gamma$.

Proof. If $\tau$ crosses $\rho$ then $\tau$ meets the triangle whose sides are $\rho, \tau_{i_{1}}$ and $\rho^{\prime}$. But $\tau$ does not cross $\tau_{i_{1}}$ since both are arcs of the triangulation $T$. Thus $\tau$ must cross $\gamma$. The proof of the second statement is similar.

Let $\mu_{j}$ denote the flip in direction $j$.

Lemma 4.3. If $S$ is simply connected, then $\gamma \in \mu_{i_{1}} \mu_{i_{2}} \ldots \mu_{i_{d}}(T)$.

Proof. We prove the statement by induction on $d$. If $d=0$ then $\gamma \in T$, and there is nothing to prove. Suppose $d>0$, then

$$
\mu_{i_{d}}(T)=T \backslash\left\{\tau_{i_{d}}\right\} \cup\left\{\tau_{i_{d}}^{\prime}\right\},
$$

and $\tau_{i_{d}}^{\prime}$ is incident to $t$, hence $\tau_{i_{d}}^{\prime}$ does not cross $\gamma$, because $S$ is simply connected. Thus $\mu_{i_{d}}(T)$ is a triangulation and $p_{1}, p_{2}, \ldots, p_{d-1}$ are its crossing points with $\gamma$ in order. By induction, $\gamma \in \mu_{i_{1}} \ldots \mu_{i_{d-1}} \mu_{i_{d}}(T)$.

Corollary 4.4. Let $S$ be simply connected. Then the arcs $\tau_{i_{1}}, \tau_{\left[\gamma_{-1}\right]}, \tau_{\left[\gamma_{0}\right]}, \rho$, and $\rho^{\prime}$ are all elements of $\mu_{i_{2}} \ldots \mu_{i_{d}}(T)$. 

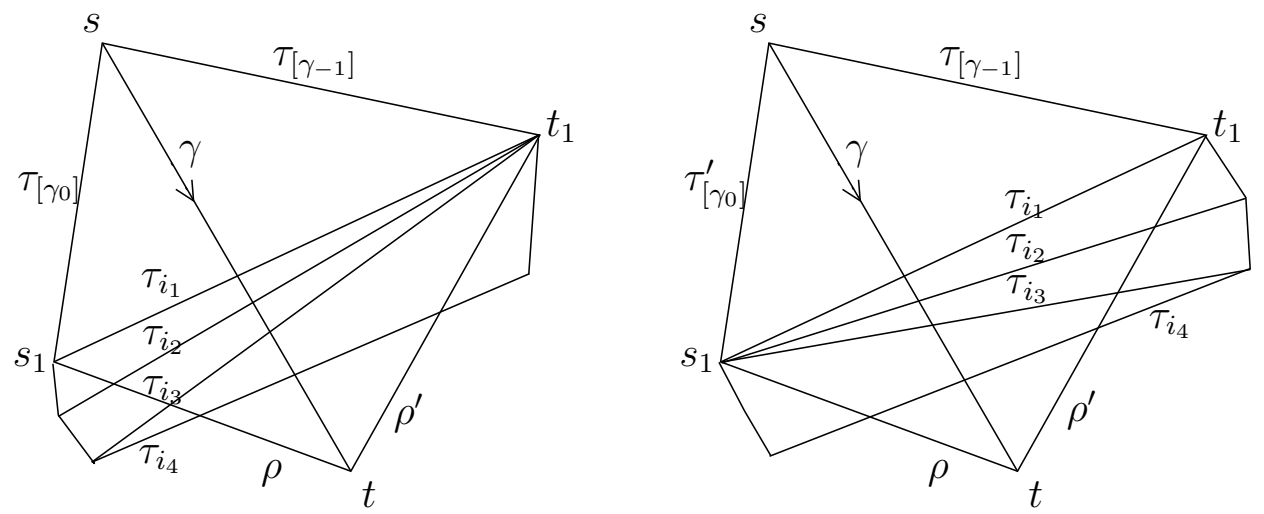

Figure 7. Two examples of triangulations. On the left $\ell=2, \ell^{\prime}=$ 4 and on the right $\ell=4, \ell^{\prime}=2$

Proof. By Lemma 4.3 we have $\gamma \in \mu_{i_{1}} \mu_{i_{2}} \ldots \mu_{i_{d}}(T)$. On the other hand, we have $\gamma \notin \mu_{i_{2}} \mu_{i_{3}} \ldots \mu_{i_{d}}(T)$ since $\tau_{i_{1}} \in \mu_{i_{2}} \mu_{i_{3}} \ldots \mu_{i_{d}}(T)$ and $\gamma$ crosses $\tau_{i_{1}}$. Hence

$$
\mu_{i_{1}} \mu_{i_{2}} \ldots \mu_{i_{d}}(T)=\mu_{i_{2}} \ldots \mu_{i_{d}}(T) \backslash\left\{\tau_{i_{1}}\right\} \cup\{\gamma\}
$$

and thus $\mu_{i_{2}} \mu_{i_{3}} \ldots \mu_{i_{d}}(T)$ contains the arcs involved in the corresponding exchange relation, namely $\tau_{\left[\gamma_{-1}\right]}, \tau_{\left[\gamma_{0}\right]}, \rho, \rho^{\prime}$.

We shall need some more notation. If $\rho \notin T$ then let $\ell$ be the least integer such that $\tau_{i_{\ell}}$ crosses $\rho$, and if $\rho \in T$ then let $\ell=d+1$. Lemma 4.2 guaranties that the integer $\ell$ is well-defined. Similarly, if $\rho^{\prime} \notin T$ then let $\ell^{\prime}$ be the least integer such that $\tau_{i_{\ell^{\prime}}}$ crosses $\rho^{\prime}$, and if $\rho^{\prime} \in T$ then let $\ell^{\prime}=d+1$, see Figure 7 Note that if $d=1$ then $\ell=\ell^{\prime}=2$, and if $d>1$ then one of $\ell, \ell^{\prime}$ is equal to 2 and the other is greater than 2. Also note that the quiver $Q_{T}$ defined in section 2.4 contains the subquiver

$$
\begin{gathered}
i_{1} \leftarrow i_{2} \leftarrow \ldots \leftarrow i_{\ell^{\prime}-1} \quad \text { if } \quad \ell=2 \\
i_{1} \rightarrow i_{2} \rightarrow \ldots \rightarrow i_{\ell-1} \text { if } \quad \ell^{\prime}=2 .
\end{gathered}
$$

Moreover, every $\tau_{i_{j}}$ with $j \geq \ell$ crosses $\rho$ and every $\tau_{i_{j}}$ with $j \geq \ell^{\prime}$ crosses $\rho^{\prime}$.

Lemma 4.5. Let $S$ be simply connected. Let $T^{\prime}=\mu_{i_{2}} \mu_{i_{3}} \ldots \mu_{i_{d}}(T)$ and let $\tilde{B}_{T^{\prime}}=$ $\left(b_{j k}^{\prime}\right)=\mu_{i_{2}} \mu_{i_{3}} \ldots \mu_{i_{d}}\left(\tilde{B}_{T}\right)$ be the $2 n \times n$ matrix obtained by applying the sequence of mutations $\mu_{i_{2}}, \mu_{i_{3}}, \ldots, \mu_{i_{d}}$ to $\tilde{B}_{T}$. Then

$$
b_{\left(n+i_{j}\right) i_{1}}^{\prime}= \begin{cases}1 & \text { if } j<\ell^{\prime} \\ 0 & \text { otherwise. }\end{cases}
$$

Proof. First note that each $b_{i j}$ is either $-1,0$ or 1 , since $S$ is simply connected. Let $Q_{T}$ be the quiver defined in section 2.4, that is, $Q_{T}$ has $2 n$ vertices labeled $1,2, \ldots, 2 n$, and there is precisely one arrow $i \rightarrow j$ whenever $\tilde{b}_{i j}=1$. Consider the 
following full subquiver of $Q_{T}$

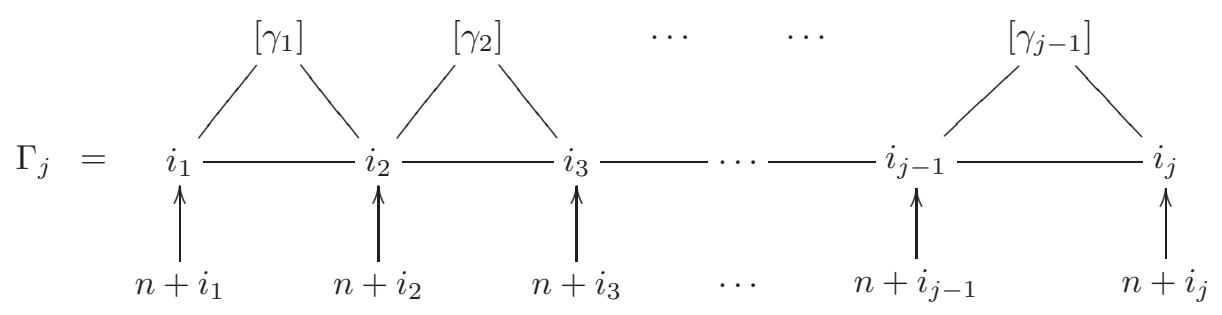

where the orientations of the edges without arrowheads is such that each triangle $i_{k}, i_{k+1},\left[\gamma_{k}\right]$ is an oriented 3 -cycle and $i_{k-1} \leftarrow i_{k}$ for all $k<\ell^{\prime}$ and $i_{\ell^{\prime}-1} \rightarrow i_{\ell^{\prime}}$. Moreover, the vertices labeled $\left[\gamma_{k}\right]$ are present only if $\tau_{\left[\gamma_{k}\right]}$ is an interior arc of $(S, M)$, thus, whenever $\tau_{\left[\gamma_{k}\right]}$ is a boundary arc in $(S, M)$, one has to remove the vertex $\left[\gamma_{k}\right]$ and all incident arrows from the above diagram. Since $S$ is simply connected, $\Gamma_{j}$ is a full subquiver of $\mu_{j+1} \ldots \mu_{d}\left(Q_{T}\right)$. Therefore, it suffices to show the following

Claim: There is precisely one arrow $\left(n+i_{j}\right) \rightarrow i_{1}$ between $\left(n+i_{j}\right)$ and $i_{1}$ in $\Gamma_{j}^{\prime}=\mu_{2} \ldots \mu_{i_{j}}\left(\Gamma_{j}\right)$ if for each $k=2, \ldots, j$ the orientation of the arrow between $i_{k-1}$ and $i_{k}$ is $i_{k-1} \leftarrow i_{k}$ in $\Gamma_{j}$; and there is no arrow between $\left(n+i_{j}\right)$ and $i_{1}$ in $\Gamma_{j}^{\prime}$ otherwise.

If the orientations of the arrow between $i_{j-1}$ and $i_{j}$ is $i_{j-1} \rightarrow i_{j}$ in $\Gamma_{j}$, then there is no arrow between $\left(n+i_{j}\right)$ and $i_{j-1}$ in the quiver $\mu_{i_{j}}\left(\Gamma_{j}\right)$, and the subsequent mutations $\mu_{i_{j-1}}, \ldots, \mu_{i_{2}}$ will not change the arrows at the vertex $\left(n+i_{j}\right)$. Thus in this case, there are no arrows between $\left(n+i_{j}\right)$ and $i_{1}$ in $\Gamma_{j}^{\prime}$. On the other hand, if the orientation of the arrow between $i_{j-1}$ and $i_{j}$ is $i_{j-1} \leftarrow i_{j}$ in $\Gamma_{j}$, then there is an arrow $\left(n+i_{j}\right) \rightarrow i_{j-1}$ in $\mu_{i_{j}}\left(\Gamma_{j}\right)$. Moreover, by deleting the vertices $i_{j},\left[\gamma_{j-1}\right]$ and $n+i_{j-1}$, we see that the quiver $\mu_{i_{j}}\left(\Gamma_{j}\right)$ contains the following quiver as a full subquiver.

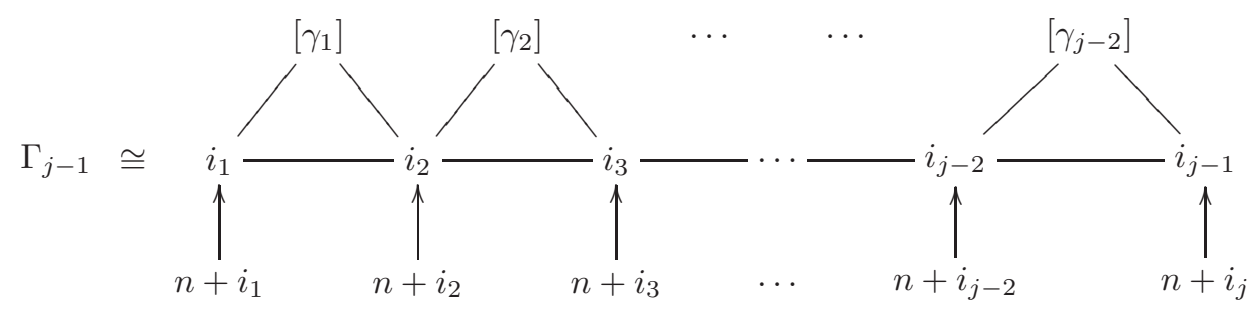

The claim now follows by induction.

We keep the setup of Lemma 4.5. Define a mapping $f$ for any complete $(T, \gamma)$ path $\alpha=\left(\alpha_{1}, \alpha_{2}, \ldots, \alpha_{2 d+1}\right)$ by

$$
f(\alpha)=\left\{\begin{array}{rrr}
\left(\alpha_{2 \ell-1}, \alpha_{2 \ell}, \ldots, \alpha_{2 d+1}\right) & \text { if } \quad \alpha_{1}=\tau_{\left[\gamma_{-1}\right]} \\
\left(\alpha_{2 \ell^{\prime}-1}, \alpha_{2 \ell^{\prime}}, \ldots, \alpha_{2 d+1}\right) & \text { if } & \alpha_{1}=\tau_{\left[\gamma_{0}\right]}
\end{array}\right.
$$

Lemma 4.6. $f$ is a bijection

$\{$ complete $(T, \gamma)$-paths $\} \stackrel{f}{\rightarrow}\{$ complete $(T, \rho)$-paths $\} \cup\left\{\right.$ complete $\left(T, \rho^{\prime}\right)$-paths $\}$, 
where $f(\alpha)$ is a complete $(T, \rho)$-path if $\alpha_{1}=\tau_{\left[\gamma_{-1}\right]}$, and $f(\alpha)$ is a complete $\left(T, \rho^{\prime}\right)$ path if $\alpha_{1}=\tau_{\left[\gamma_{0}\right]}$. Moreover

$$
\left.\begin{array}{rl}
\frac{x_{\tau_{[\gamma-1]}}}{x_{i_{1}}} x(f(\alpha)) & =x(\alpha) \\
y(f(\alpha)) & =y(\alpha)
\end{array}\right\} \quad \text { if } \alpha_{1}=\tau_{\left[\gamma_{-1}\right]}
$$

where $Y^{\prime}=y_{i_{1}} y_{i_{2}} \ldots y_{i_{\ell^{\prime}-1}}$.

Proof. First we show that $f$ is well defined. Clearly, $f(\alpha)$ is a $T$-path. If $\alpha_{1}=\tau_{\left[\gamma_{-1}\right]}$, then the even $\operatorname{arcs} \tau_{i_{\ell}}, \tau_{i_{\ell+1}}, \ldots, \tau_{i_{d}}$ of $f(\alpha)$ are crossing $\rho$ in order, and, by Lemma 4.2, $\rho$ does not cross any other $\operatorname{arcs}$ of $T$. On the other hand, if $\alpha_{1}=\tau_{\left[\gamma_{0}\right]}$, then the even $\operatorname{arcs} \tau_{i_{\ell^{\prime}}}, \tau_{i_{\ell^{\prime}+1}}, \ldots, \tau_{i_{d}}$ of $f(\alpha)$ are crossing $\rho^{\prime}$ in order, and, again by Lemma 4.2. $\rho^{\prime}$ does not cross any other arcs of $T$. This shows that $f(\alpha)$ satisfies axiom (T1). The axiom (T2) follows from Lemma 4.1 (b), and therefore, $f$ is well defined.

Next we show that $f$ is injective. Suppose $f(\alpha)=f\left(\alpha^{\prime}\right)$. Then it follows from the definition of $f$ that $\alpha_{1}=\alpha_{1}^{\prime}$ and $\alpha_{2}=\alpha_{2}^{\prime}$. Suppose first that $\alpha_{1}=\tau_{\left[\gamma_{-1}\right]}$, see Figure 7. If $\ell=2$ then $f(\alpha)=\left(\alpha_{3}, \alpha_{4}, \ldots, \alpha_{2 d+1}\right)$, while $f\left(\alpha^{\prime}\right)=\left(\alpha_{3}^{\prime}, \alpha_{4}^{\prime}, \ldots, \alpha_{2 d+1}^{\prime}\right)$, and thus $\alpha=\alpha^{\prime}$. Otherwise, $\ell^{\prime}=2$ and since the even $\operatorname{arcs}$ of $\alpha$ and $\alpha^{\prime}$ must cross $\gamma$ in order, there is only one possibility for the first $2 \ell-2 \operatorname{arcs}$ in $\alpha$ and $\alpha^{\prime}$, namely $\alpha_{1}=\alpha_{1}^{\prime}=\tau_{\left[\gamma_{-1}\right]}, \alpha_{2}=\alpha_{2}^{\prime}=\tau_{i_{1}}$ and $\alpha_{2 j-1}=\alpha_{2 j}=\tau_{i_{j}}=\alpha_{2 j-1}^{\prime}=\alpha_{2 j}^{\prime}$ for $j=2,3, \ldots, \ell-1$, see the right hand side of Figure 7 Hence $\alpha=\alpha^{\prime}$. Suppose now that $\alpha_{1}=\tau_{\left[\gamma_{0}\right]}$. If $\ell^{\prime}=2$ then clearly $\alpha=\alpha^{\prime}$. Otherwise, there is only one possibility for the first $2 \ell^{\prime}-2$ arcs in $\alpha$ and $\alpha^{\prime}$, namely $\alpha_{2 j-1}=\alpha_{2 j}=\tau_{i_{j}}$ and $\alpha_{2 j-1}^{\prime}=\alpha_{2 j}^{\prime}=\tau_{i_{j}}$ for $j=2,3, \ldots, \ell^{\prime}-1$, see the left hand side of Figure 7 Hence $\alpha=\alpha^{\prime}$.

It remains to show that $f$ is surjective. Let $\hat{\alpha}=\left(\alpha_{2 \ell-1}, \alpha_{2 \ell}, \ldots, \alpha_{2 d+1}\right)$ be a complete $(T, \rho)$-path. Then let

$$
\alpha= \begin{cases}\left(\tau_{\left[\gamma_{-1}\right]}, \tau_{i_{1}}, \alpha_{2 \ell-1}, \alpha_{2 \ell}, \ldots, \alpha_{2 d+1}\right) & \text { if } \ell=2 \\ \left(\tau_{\left[\gamma_{-1}\right]}, \tau_{i_{1}}, \tau_{i_{2}}, \tau_{i_{2}}, \tau_{i_{3}}, \tau_{i_{3}}, \ldots, \tau_{i_{\ell-1}}, \tau_{i_{\ell-1}}, \alpha_{2 \ell-1}, \alpha_{2 \ell}, \ldots, \alpha_{2 d+1}\right) & \text { if } \ell>2 .\end{cases}
$$

Then $\alpha$ is a $T$-path, its even arcs are $\tau_{i_{1}}, \tau_{i_{2}}, \ldots, \tau_{i_{d}}$ in order, and then it follows from Lemma 4.1 (b) that $\alpha$ is a complete $(T, \gamma)$-path. Moreover, $f(\alpha)=\hat{\alpha}$. On the other hand, let $\check{\alpha}=\left(\alpha_{2 \ell^{\prime}-1}, \alpha_{2 \ell^{\prime}}, \ldots, \alpha_{2 d+1}\right)$ be a complete $\left(T, \rho^{\prime}\right)$-path. Then let

$\alpha= \begin{cases}\left(\tau_{\left[\gamma_{0}\right]}, \tau_{i_{1}}, \alpha_{2 \ell^{\prime}-1}, \alpha_{2 \ell^{\prime}}, \ldots, \alpha_{2 d+1}\right) & \text { if } \ell^{\prime}=2 \\ \left(\tau_{\left[\gamma_{0}\right]}, \tau_{i_{1}}, \tau_{i_{2}}, \tau_{i_{2}}, \tau_{i_{3}}, \tau_{i_{3}}, \ldots, \tau_{i_{\ell^{\prime}-1}}, \tau_{i_{\ell^{\prime}-1}}, \alpha_{2 \ell^{\prime}-1}, \alpha_{2 \ell^{\prime}}, \ldots, \alpha_{2 d+1}\right) & \text { if } \ell^{\prime}>2 .\end{cases}$

Then $\alpha$ is a complete $(T, \gamma)$-path, and $f(\alpha)=\check{\alpha}$. Thus $f$ is surjective, and hence a bijection.

Now we show the identities in the statement of Lemma 4.6 Suppose first that $\alpha_{1}=\tau_{\left[\gamma_{-1}\right]}$. Then $\alpha_{2}=\tau_{i_{1}}$ is not $\gamma$-oriented. If $\ell=2$ then $f(\alpha)=$ 
$\left(\alpha_{3}, \alpha_{4}, \ldots, \alpha_{2 d+1}\right)$ and

$$
x(\alpha)=\frac{x_{\left.\tau_{[\gamma-1}\right]}}{x_{i_{1}}} x(f(\alpha)) \quad \text { and } \quad y(\alpha)=y(f(\alpha)) .
$$

On the other hand, if $\ell \neq 2$ then

$$
\alpha=\left(\tau_{[\gamma-1]}, \tau_{i_{1}}, \tau_{i_{2}}, \tau_{i_{2}}, \ldots, \tau_{i_{\ell-1}}, \tau_{i_{\ell-1}}, \alpha_{2 \ell-1}, \alpha_{2 \ell}, \ldots\right)
$$

where none of the first $\ell-1$ even arcs is $\gamma$-oriented, see the right hand side of Figure 77, and $f(\alpha)=\left(\alpha_{2 \ell-1}, \alpha_{2 \ell}, \ldots\right)$. Hence

$$
x(\alpha)=\frac{x_{\tau_{[\gamma-1]}}}{x_{i_{1}}} x(f(\alpha)) \quad \text { and } \quad y(\alpha)=y(f(\alpha)) .
$$

This proves the lemma in the case $\alpha_{1}=\tau_{\left[\gamma_{-1}\right]}$.

Suppose now that $\alpha_{1}=\tau_{\left[\gamma_{0}\right]}$. Then $\alpha_{2}$ is $\gamma$-oriented. If $\ell^{\prime}=2$ then $f(\alpha)=$ $\left(\alpha_{3}, \alpha_{4}, \ldots, \alpha_{2 d+1}\right)$ and

$$
x(\alpha)=\frac{x_{\tau_{\left[\gamma_{0}\right]}}}{x_{i_{1}}} x(f(\alpha)) \quad \text { and } \quad y(\alpha)=y_{i_{1}} y(f(\alpha))=Y^{\prime} y(f(\alpha)) .
$$

On the other hand, if $\ell^{\prime} \neq 2$ then

$$
\alpha=\left(\tau_{\left[\gamma_{0}\right]}, \tau_{i_{1}}, \tau_{i_{2}}, \tau_{i_{2}}, \ldots, \tau_{i_{\ell^{\prime}-1}}, \tau_{i_{\ell^{\prime}-1}}, \alpha_{2 \ell^{\prime}-1}, \alpha_{2 \ell^{\prime}}, \ldots\right)
$$

where each of the first $\ell^{\prime}-1$ even arcs is $\gamma$-oriented, see the left hand side of Figure 7, and $f(\alpha)=\left(\alpha_{2 \ell^{\prime}-1}, \alpha_{2 \ell^{\prime}}, \ldots\right)$. Hence

$$
x(\alpha)=\frac{x_{\tau_{\left[\gamma_{0}\right]}}}{x_{i_{1}}} x(f(\alpha)) \quad \text { and } \quad y(\alpha)=y_{i_{1}} y_{i_{2}} \ldots y_{i_{\ell^{\prime}-1}} y(f(\alpha))=Y^{\prime} y(f(\alpha)) .
$$

Proof of Theorem 3.2 in the simply connected case. We prove the Theorem by induction on $d$. If $d=0$ then $\gamma \in T$ and the only complete $(T, \gamma)$-path is the path $\alpha=(\gamma)$. Hence $\sum_{\alpha} x(\alpha) y(\alpha)=x_{\gamma}$, as desired. Suppose now $d \geq 1$. By Corollary 4.4 the triangulation $T^{\prime}=\mu_{i_{2}} \mu_{i_{3}} \ldots \mu_{i_{d}}(T)$ contains $\tau_{\left[\gamma_{-1}\right]}, \tau_{\left[\gamma_{0}\right]}, \rho, \rho^{\prime}$, and $\tau_{i_{1}}$, and we thus have the exchange relation

$$
x_{i_{1}} x_{\gamma}=Y x_{\left.\tau_{[\gamma-1]}\right]} x_{\rho}+Y^{\prime} x_{\tau_{\left[\gamma_{0}\right]}} x_{\rho^{\prime}}
$$

where

$$
Y=\prod_{b_{\left(n+i_{j}\right) i_{1}}^{\prime} \leq 0} y_{i_{j}}^{-b_{\left(n+i_{j}\right) i_{1}}^{\prime}} \quad \text { and } \quad Y^{\prime}=\prod_{b_{\left(n+i_{j}\right) i_{1}}^{\prime} \geq 0} y_{i_{j}}^{b_{\left(n+i_{j}\right) i_{1}}^{\prime}}
$$

and where $B^{\prime}=\left(b_{i j}^{\prime}\right)$ is the $2 n \times n$ matrix given by $B^{\prime}=\mu_{i_{2}} \ldots \mu_{i_{d}}\left(B_{T}\right)$. By Lemma 4.5, we have $b_{\left(n+i_{j}\right) i_{1}}^{\prime}=1$ if $j<\ell^{\prime}$ and $b_{\left(n+i_{j}\right) i_{1}}^{\prime}=0$ otherwise. In particular, $Y=1$; and $Y^{\prime}=y_{i_{1}} y_{i_{2}} \cdots y_{i_{\ell^{\prime}-1}}$ as in Lemma 4.6 .

The number of crossings between $\gamma$ and $T$ is strictly larger than the number of crossings between $\rho$ and $T$ and strictly larger than the number of crossings between $\rho^{\prime}$ and $T$. Hence, by induction, equation (8) implies

$$
x_{\gamma}=\sum_{\beta} \frac{x_{\tau_{[\gamma-1]}}}{x_{i_{1}}} x(\beta) y(\beta)+\sum_{\beta^{\prime}} \frac{x_{\tau_{\left[\gamma_{0}\right]}}}{x_{i_{1}}} x\left(\beta^{\prime}\right) Y^{\prime} y\left(\beta^{\prime}\right),
$$

where, in the first sum, $\beta$ runs over all complete $(T, \rho)$-paths in $(S, M)$, and, in the second sum, $\beta^{\prime}$ runs over all complete $\left(T, \rho^{\prime}\right)$-paths in $(S, M)$. 
Applying Lemma 4.6 to equation (9) we get

$$
x_{\gamma}=\sum_{\alpha: \alpha_{1}=\tau_{[\gamma-1]}} x(\alpha) y(\alpha)+\sum_{\alpha: \alpha_{1}=\tau_{\left[\gamma_{0}\right]}} x(\alpha) y(\alpha),
$$

where, in the first sum, $\alpha$ runs over all complete $(T, \gamma)$-paths starting with $\tau_{\left[\gamma_{-1}\right]}$, and, in the second sum, $\alpha$ runs over all $(T, \gamma)$-paths starting with $\tau_{\left[\gamma_{0}\right]}$.

Since $S$ is simply connected and $\alpha_{2}=\tau_{i_{1}}$ for any complete $(T, \gamma)$-path $\alpha$, Lemma 4.1 (b) implies that we must have $\alpha_{1}=\tau_{\left[\gamma_{-1}\right]}$ or $\alpha_{1}=\tau_{\left[\gamma_{0}\right]}$ for any complete $(T, \gamma)$ path $\alpha$.

Therefore the right hand side of equation (10) equals the right hand side of the formula in Theorem 3.2. This proves the theorem in the case where $S$ is simply connected. The general case will be proved in section 5.5

\section{UNIVERSAL COVER}

In this section, we will use covering techniques to prove Theorem 3.2 in the general case. The idea of the proof is to work in the universal cover of $S$ in which we can apply the Theorem 3.2 for simply connected surfaces, and then project the results to the original surface $S$. First we need to investigate Galois coverings of quivers.

5.1. Galois coverings. Let $k$ be a field. Any quiver $Q$ can be considered as a $k$-category, whose objects are the vertices of $Q$ and for two vertices $x, y$ of $Q$, the space $\operatorname{Hom}_{Q}(x, y)$ is spanned by the set of arrows from $x$ to $y$ in $Q$. The composition of morphisms is the composition of arrows.

Let $Q, \tilde{Q}$ be two quivers and $\pi: \tilde{Q} \rightarrow Q$ a functor, that is, $\pi$ maps vertices to vertices and arrows to arrows respecting the composition. Let $\operatorname{Aut}(\tilde{Q})$ be the group of autoequivalences of $\tilde{Q}$, and let $G$ be a subgroup of $\operatorname{Aut}(\tilde{Q})$ whose action on the objects of $\tilde{Q}$ is free (that is $g(x) \neq x$ for all vertices $x$ and all $g \in G, g \neq i d$ ) and such that $\pi \circ g=\pi$ for all $g \in G$.

Then $\pi: \tilde{Q} \rightarrow Q$ is called a Galois covering with group $G$ if the orbit category $\tilde{Q} / G$ of $\tilde{Q}$ under the action of $G$ is equivalent to $Q$.

If $j$ is a vertex of a quiver $Q$, let $\mu_{j}$ denote the mutation in direction $j$. Let $\pi: \tilde{Q} \rightarrow Q$ be a Galois covering of quivers with group $G$. The elements of the fiber $\pi^{-1}(j)$ of $j$ in $\tilde{Q}$ are of the form $j_{g}$ with $g \in G$. Let $\tilde{\mu}_{j}$ denote the infinite sequence of mutations $\left(\mu_{j_{g}}\right)_{g \in G}$.

Lemma 5.1. If $\pi: \tilde{Q} \rightarrow Q$ is a Galois covering of quivers with group $G$, then there exists a Galois covering $\pi^{\prime}: \tilde{\mu}_{j}(\tilde{Q}) \rightarrow \mu_{j}(Q)$ of the mutated quivers with the same group $G$.

Proof. The mutated quiver $\mu_{j}(Q)$ is obtained from $Q$ by the following operations:

(1) For every path $i \stackrel{\alpha}{\rightarrow} j \stackrel{\beta}{\rightarrow} k$ introduce a new arrow $i \stackrel{[\alpha \beta]}{\rightarrow} k$.

(2) Replace each arrow $\alpha$ starting at (respectively ending at) $j$ by its opposite $\alpha^{*}$ ending at (respectively starting at) $j$.

(3) Cancel all 2-cycles.

For each arrow $i \stackrel{\alpha}{\rightarrow} j$ (respectively $j \stackrel{\beta}{\rightarrow} k$ ) and for each $g \in G$ there exists a unique arrow $i_{h} \stackrel{\alpha_{g}}{\rightarrow} j_{g}$ (respectively $j_{g} \stackrel{\beta_{g}}{\rightarrow} k_{h^{\prime}}$ ) that ends at $j_{g}$ (respectively starts at $j_{g}$ ) for some $h, h^{\prime} \in G$, and such that $\pi\left(\alpha_{g}\right)=\alpha$. 
Thus the mutated quiver $\tilde{\mu}_{j}(\tilde{Q})$ is obtained from $\tilde{Q}$ by the following operations:

(1) For every path $i_{h} \stackrel{\alpha_{g}}{\rightarrow} j_{g} \stackrel{\beta_{g}}{\rightarrow} k_{h^{\prime}}$ with $h, g, h^{\prime} \in G$ introduce a new arrow $i_{h} \stackrel{[\alpha \beta]_{g}}{\longrightarrow} k_{h^{\prime}}$.

(2) Replace each arrow $\alpha_{g}$ starting at (respectively ending at) $j_{g}$ by its opposite $\alpha_{g}^{*}$ ending at (respectively starting at) $j_{g}$.

(3) Cancel all 2-cycles.

Define $\pi^{\prime}: \tilde{\mu}_{j}(\tilde{Q}) \rightarrow \mu_{j}(Q)$ as follows. Let $\pi^{\prime}=\pi$ on all vertices of $\tilde{\mu}_{j} \tilde{Q}$ and let $\pi^{\prime}=\pi$ on those arrows that lie in both $\tilde{\mu}_{j}(\tilde{Q})$ and $\tilde{Q}$; and let $\pi^{\prime}\left([\alpha \beta]_{g}\right)=[\alpha \beta]$ and $\pi^{\prime}\left(\alpha_{g}^{*}\right)=\alpha^{*}$. The group $G$ acts on the vertices of $\tilde{\mu}_{j}(\tilde{Q})$ in the same way as on $\tilde{Q}$. Its action on the arrows of $\tilde{\mu}_{j}(\tilde{Q})$ that have not been modified by the mutation $\tilde{\mu}_{j}$ is the same as on $\tilde{Q}$. On the new arrows $[\alpha \beta]_{g}$, the action of an element $g^{\prime} \in G$ is given by

$$
g^{\prime}\left([\alpha \beta]_{g}\right)=\left(i_{g^{\prime} h} \stackrel{[\alpha \beta]_{g^{\prime} g}}{\longrightarrow} k_{g^{\prime} h}\right),
$$

for all $g, g^{\prime}, h, h^{\prime} \in G$. Clearly, $\pi^{\prime} \circ g=\pi^{\prime}$ for all $g \in G$, and thus $\pi^{\prime}$ is a Galois covering of quivers with the same group as $\pi$.

5.2. Triangulations and quivers. Let $\pi: \tilde{S} \rightarrow S$ be a universal cover of the surface $S$, and let $\tilde{M}=\pi^{-1}(M)$ and $\tilde{T}=\pi^{-1}(T)$.

From the theory of covering spaces, we know that for each point $p \in S$ the fiber $\pi^{-1}(p)$ is the fundamental group $\Pi_{1}(S)$ of the surface $S$. Hence $\pi^{-1}(p)=\left\{\tilde{p}_{\sigma} \mid \sigma \in\right.$ $\left.\Pi_{1}(S)\right\}$. Furthermore, for each path $\tau$ in $S$ with starting point $p \in S$ and for each $\sigma \in \Pi_{1}(S)$ there is a unique lift $\tilde{\tau}_{\sigma}$ in $\tilde{S}$ with starting point $\tilde{p}_{\sigma}$ in the fiber of $p$. The triangulation $\tilde{T}$ consists of $\operatorname{arcs} \tilde{\tau}_{\sigma}$ where $\sigma \in \Pi_{1}(S)$ and $\pi\left(\tilde{\tau}_{\sigma}\right) \in T$.

Let $Q_{T}$ be the quiver associated to the triangulation $T$ as in section 2.4 Recall that $Q_{T}$ has $2 n$ vertices labeled $1,2, \ldots, 2 n$, where the first $n$ vertices correspond to the interior arcs of the triangulation $T$, and the vertex $n+i$ correspond to the coefficient $y_{i}$, for $i=1,2, \ldots, n$. Since the cluster algebra $\mathcal{A}$ has principal coefficients in the initial seed, we have an arrow $(n+i) \rightarrow j$ in $Q_{T}$ if $i=j$ and there are no arrows between $(n+i)$ and $j$ in $Q_{T}$ if $i \neq j$.

Let $Q_{\tilde{T}}$ be the (infinite) quiver associated in the same way to triangulation $\tilde{T}$ of $(\tilde{S}, \tilde{M})$.

The vertices of the quiver $Q_{\tilde{T}}$ are labeled by tuples $(\tau, \sigma)$ where $\tau \in\{1,2, \ldots, 2 n\}$ is a vertex of $Q_{T}$ and $\sigma \in \Pi_{1}(S)$, and the arrows by $\alpha_{\sigma}$ where $\alpha$ is an arrow in $Q_{T}$ and $\sigma \in \Pi_{1}(S)$. Hence $\pi$ induces a functor $\pi: Q_{\tilde{T}} \rightarrow Q_{T}$. The fundamental group $\Pi_{1}(S)$ acts on $Q_{\tilde{T}}$ by autoequivalences as $\rho \cdot(\tau, \sigma)=(\tau, \rho \sigma)$, for all $\tau \in\{1,2, \ldots, 2 n\}$, and all $\sigma, \rho \in \Pi_{1}(S)$; and $\rho \cdot \alpha_{\sigma}=\alpha_{\rho \sigma}$, for all arrows $\alpha$ in $Q_{T}$, and all $\sigma, \rho \in \Pi_{1}(S)$. This action is free on vertices and for all $\sigma \in \Pi_{1}(S)$, we have $\pi \circ \sigma=\pi$. Moreover, $Q_{T} \cong Q_{\tilde{T}} / \Pi_{1}(S)$, and thus $\pi: Q_{\tilde{T}} \rightarrow Q_{T}$ is a Galois covering of quivers with group $\Pi_{1}(S)$. Applying Lemma 5.1, we get the following theorem.

Theorem 5.2. Let $T^{\prime}$ be the triangulation of $(S, M)$ obtained from $T$ by a sequence of mutations $T^{\prime}=\mu_{j_{1}} \mu_{j_{2}} \ldots \mu_{j_{s}}(T)$. Let $\tilde{T}=\pi^{-1}(T)$ be the lifted triangulation of $(\tilde{S}, \tilde{M})$ and denote by $Q_{\tilde{T}}$ the corresponding quiver. Let $\tilde{T}^{\prime}=\tilde{\mu}_{j_{1}} \tilde{\mu}_{j_{2}} \ldots \tilde{\mu}_{j_{s}}(\tilde{T})$ and $Q_{\tilde{T}^{\prime}}=\tilde{\mu}_{j_{1}} \tilde{\mu}_{j_{2}} \ldots \tilde{\mu}_{j_{s}}\left(Q_{\tilde{T}}\right)$. Then $T^{\prime}=\pi\left(\tilde{T}^{\prime}\right)$ and $\pi: Q_{\tilde{T}^{\prime}} \rightarrow Q_{T^{\prime}}$ is a Galois covering of quivers with group $\Pi_{1}(S)$. 
5.3. Cluster algebras. In this subsection, we will use the universal cover to define a new cluster algebra $\tilde{\mathcal{A}}$ in which we can use Theorem 3.2 for simply connected surfaces. We will not work inside the universal covering space $\tilde{S}$ itself but we will restrict to a subsurface of $S$ that contains a lift of the $\operatorname{arc} \gamma$ and has a finite triangulation. We also need a special coefficient system that takes care of the principal coefficients as well as of the boundary of the surface.

We keep the setup of section 3 . Let $\pi: \tilde{S} \rightarrow S$ be a universal cover of the surface $S$, and let $\tilde{M}=\pi^{-1}(M)$ and $\tilde{T}=\pi^{-1}(T)$.

Choose a point $\tilde{s}$ in the fiber $\pi^{-1}(s)$ of the starting point $s$ of the $\operatorname{arc} \gamma$. There exists a unique lift $\tilde{\gamma}$ of $\gamma$ starting at $\tilde{s}$. Then $\tilde{\gamma}$ is the concatenation of subpaths $\tilde{\gamma}_{0}, \tilde{\gamma}_{1}, \ldots, \tilde{\gamma}_{d}$ where $\tilde{\gamma}_{k}$ is a path from a point $\tilde{p}_{k}$ to a point $\tilde{p}_{k+1}$ such that $\tilde{\gamma}_{k}$ is a lift of $\gamma_{k}$ and $\tilde{p}_{k} \in \pi^{-1}\left(p_{k}\right)$, for $k=0,1, \ldots, d$. Let $\tilde{t}=\tilde{p}_{d+1} \in \pi^{-1}(t)$.

For $k$ from 1 to $d$, let $\tilde{\tau}_{i_{k}}$ be the unique lift of $\tau_{i_{k}}$ running through $\tilde{p}_{k}$. For $k$ from 1 to $d-1$ let $\tilde{\tau}_{\left[\gamma_{k}\right]}$ be the unique lift of $\tau_{\left[\gamma_{k}\right]}$ that is bounding a triangle $\tilde{\Delta}_{k}$ in $\tilde{T}$ with $\tilde{\tau}_{i_{k}}$ and $\tilde{\tau}_{i_{k+1}}$. Let $\tilde{\tau}_{\left[\gamma_{-1}\right]}$ and $\tilde{\tau}_{\left[\gamma_{0}\right]}$ be the unique lifts of $\tau_{\left[\gamma_{-1}\right]}$ and $\tau_{\left[\gamma_{0}\right]}$ that, together with $\tilde{\tau}_{i_{1}}$, are bounding a triangle $\tilde{\Delta}_{0}$, and let $\tilde{\tau}_{\left[\gamma_{d}\right]}$ and $\tilde{\tau}_{\left[\gamma_{d+1}\right]}$ be the unique lifts of $\tau_{\left[\gamma_{d}\right]}$ and $\tau_{\left[\gamma_{d+1}\right]}$ that, together with $\tilde{\tau}_{i_{d}}$, are bounding a triangle $\tilde{\Delta}_{d}$.

Definition 3. Let $\tilde{S}(\gamma) \subset \tilde{S}$ be the union of the d triangles $\tilde{\Delta}_{0}, \tilde{\Delta}_{1}, \ldots, \tilde{\Delta}_{d}$ and let $\tilde{M}(\gamma)=\tilde{M} \cap \tilde{S}(\gamma)$ and $\tilde{T}(\gamma)=\tilde{T} \cap \tilde{S}(\gamma)$.

Proposition 5.3. $(\tilde{S}(\gamma), \tilde{M}(\gamma))$ is a simply connected unpunctured surface of which $\tilde{T}(\gamma)$ is a triangulation. This triangulation $\tilde{T}(\gamma)$ consists of $d$ interior arcs and $d+3$ boundary arcs.

In particular, each triangle $\tilde{\Delta}_{k}$ in $\tilde{T}(\gamma)$ contains a boundary arc.

Proof. This follows immediately from the construction.

Associate a quiver $\tilde{Q}_{\tilde{T}(\gamma)}$ to $\tilde{T}(\gamma)$ as follows: The set of vertices of $\tilde{Q}_{\tilde{T}(\gamma)}$ is

$$
\left\{i_{1}, i_{2}, \ldots, i_{d}, c_{1}, c_{2}, \ldots, c_{d},\left[\gamma_{-1}\right],\left[\gamma_{0}\right], \ldots,\left[\gamma_{d+1}\right]\right\}
$$

corresponding to the $d$ interior $\operatorname{arcs} \tilde{\tau}_{i_{1}}, \tilde{\tau}_{i_{2}}, \ldots, \tilde{\tau}_{i_{d}}$ of $\tilde{T}(\gamma)$, the $d+3$ boundary $\operatorname{arcs} \tilde{\tau}_{\left[\gamma_{-1}\right]}, \tilde{\tau}_{\left[\gamma_{0}\right]}, \ldots, \tilde{\tau}_{\left[\gamma_{d+1}\right]}$ of $\tilde{T}(\gamma)$, and one extra vertex $c_{j}$ for each interior $\operatorname{arc} \tilde{\tau}_{i_{j}}$. The set of arrows consists of one arrow $c_{j} \rightarrow i_{j}$, for each $j, 1 \leq j \leq d$; and one arrow $i_{j} \rightarrow i_{k}$ (respectively $i_{j} \rightarrow\left[\gamma_{\ell}\right] ;\left[\gamma_{\ell}\right] \rightarrow i_{j}$ ) whenever $\left(\tilde{\tau}_{i_{j}}, \tilde{\tau}_{i_{k}}\right.$ ) (respectively $\left.\left(\tilde{\tau}_{i_{j}}, \tilde{\tau}_{\left[\gamma_{\ell}\right]}\right)\right)$ are sides of the same triangle in $\tilde{T}(\gamma)$ with $\tilde{\tau}_{i_{k}}$ following $\tilde{\tau}_{i_{j}}$ (respectively $\tilde{\tau}_{\left[\gamma_{\ell}\right]}$ following $\tilde{\tau}_{i_{j}} ; \tilde{\tau}_{i_{j}}$ following $\left.\tilde{\tau}_{\left[\gamma_{\ell}\right]}\right)$ in the counter-clockwise order. Let us point out that the difference between this construction and the one in section 2.4 is that it involves also the boundary arcs of the surface.

Since each of the triangles in $\tilde{S}(\gamma)$ is a lift of an oriented triangle in $S$, the orientation of $S$ lifts to an orientation of $\tilde{S}(\gamma)$ and we have

Lemma 5.4. A path $\tilde{\alpha}_{2 k}$ along the arc $\tilde{\tau}_{i_{k}}$ is $\tilde{\gamma}$-oriented if and only if $\pi\left(\tilde{\alpha}_{2 k}\right)$ along the arc $\tau_{i_{k}}$ is $\gamma$-oriented.

Let

$$
\tilde{\mathbb{P}}=\operatorname{Trop}\left(u_{i_{1}}, u_{i_{2}}, \ldots, u_{i_{d}}, u_{\left[\gamma_{-1}\right]}, u_{\left[\gamma_{0}\right]}, \ldots, u_{\left[\gamma_{d+1}\right]}\right)
$$

be the tropical semifield on $2 d+3$ generators, where the first $d$ generators correspond to the $d$ interior arcs $\tilde{\tau}_{i_{k}}, k=1,2, \ldots, d$ and the last $d+3$ generators $u_{\left[\gamma_{j}\right]}$ correspond to the $d+3$ boundary $\operatorname{arcs} \tilde{\tau}_{\left[\gamma_{j}\right]}, j=-1,0, \ldots, d+1$. Let $\tilde{\mathcal{F}}$ be the field of rational functions in $d$ variables with coefficients in $\mathbb{Q} \tilde{\mathbb{P}}$. 
Definition 4. Let $\tilde{\mathcal{A}}=\mathcal{A}(\tilde{\mathbf{x}}, \tilde{\mathbf{y}}, \tilde{B})$ be the cluster algebra in $\tilde{\mathcal{F}}$ given by the initial seed $\tilde{\Sigma}$ :

$$
\begin{aligned}
\tilde{\mathbf{x}}=\left\{\tilde{x}_{i_{1}}, \ldots, \tilde{x}_{i_{d}}\right\} & \text { where } \tilde{x}_{i_{k}}=x_{\tilde{\tau}_{i_{k}}} \\
\tilde{\mathbf{y}}=\left\{\tilde{y}_{i_{1}}, \ldots, \tilde{y}_{i_{d}}\right\} & \text { where } \tilde{y}_{i_{k}}=u_{i_{k}} \prod_{\left[\gamma_{j}\right] \rightarrow i_{k} \in \tilde{Q}_{\tilde{T}(\gamma)}} u_{\left[\gamma_{j}\right]} \prod_{\left[\gamma_{j}\right] \leftarrow i_{k} \in \tilde{Q}_{\tilde{T}(\gamma)}} u_{\left[\gamma_{j}\right]}^{-1} \\
\tilde{B}=B_{\tilde{T}(\gamma)} &
\end{aligned}
$$

Thus the cluster algebra has coefficients combined from principal coefficients, the $u_{i_{k}}$ term, and boundary coefficients, the $u_{\left[\gamma_{j}\right]}$ terms coming from boundary arcs.

For each $k=1,2, \ldots, d$, denote by $\tilde{x}_{i_{k}}^{\prime}$ the cluster variable obtained by mutation in direction $k$. That is $\mu_{k}(\tilde{\mathbf{x}})=\tilde{\mathbf{x}} \backslash\left\{\tilde{x}_{i_{k}}\right\} \cup\left\{\tilde{x}_{i_{k}}^{\prime}\right\}$.

Proposition 5.5. The cluster algebra $\tilde{\mathcal{A}}$ is an acyclic cluster algebra of finite type $A_{d}$ with acyclic seed $\tilde{\Sigma}$. In particular, $\tilde{\mathcal{A}}$ is generated over $\mathbb{Z} \tilde{\mathbb{P}}$ by the $2 d$ cluster variables

$$
\tilde{x}_{i_{1}}, \tilde{x}_{i_{2}}, \ldots, \tilde{x}_{i_{d}}, \tilde{x}_{i_{1}}^{\prime}, \tilde{x}_{i_{2}}^{\prime}, \ldots, \tilde{x}_{i_{d}}^{\prime} .
$$

Proof. The surface $\tilde{S}(\gamma)$ is topologically a polygon with $d+3$ vertices, which implies that the cluster algebra is of type $A_{d}$. The seed $\tilde{\Sigma}$ is acyclic since, by Proposition 5.3. each triangle in $\tilde{T}(\gamma)$ has at least one side given by a boundary arc. The last statement now follows from [BFZ, Cor 1.21].

The following Lemma is shown in $\underline{\mathrm{ST}}$. It will allow us to use induction later.

Lemma 5.6. [ST, Lemma 4.6] Let $T$ be a triangulation of an unpunctured surface $(S, M)$, and let $\beta$ be an arc in $S$ which is not in $T$. Let $k$ be the number of crossings between $\beta$ and $T$. Then there exist five arcs $\rho_{1}, \rho_{2}, \sigma_{1}, \sigma_{2}$ and $\beta^{\prime}$ in $S$ such that

(a) each of $\rho_{1}, \rho_{2}, \sigma_{1}, \sigma_{2}$ and $\beta^{\prime}$ crosses $T$ less than $k$ times,

(b) $\rho_{1}, \rho_{2}, \sigma_{1}, \sigma_{2}$ are the sides of a simply connected quadrilateral $V$ in which $\beta$ and $\beta^{\prime}$ are the diagonals.

Theorem 5.7. The universal cover $\pi: \tilde{S} \rightarrow S$ induces a homomorphism of algebras $\pi_{*}: \tilde{\mathcal{A}} \rightarrow \mathcal{A}$ defined by $\pi_{*}\left(\tilde{x}_{i_{k}}\right)=x_{i_{k}}, \pi_{*}\left(\tilde{x}_{i_{k}}^{\prime}\right)=x_{i_{k}}^{\prime}, \pi_{*}\left(u_{i_{k}}\right)=y_{i_{k}}$ and $\pi_{*}\left(u_{\left[\gamma_{j}\right]}\right)=$ $x_{\left[\gamma_{j}\right]}$. Moreover, if $\tilde{\beta}$ is an interior arc in $(\tilde{S}(\gamma), \tilde{M}(\gamma))$ which is a lift of an arc $\beta$ in $(S, M)$, then $\pi_{*}\left(\tilde{x}_{\tilde{\beta}}\right)=x_{\beta}$.

Proof. By Proposition 5.5, $\tilde{\mathcal{A}}$ is generated over $\mathbb{Z} \tilde{\mathbb{P}}$ by the elements $\tilde{x}_{i_{k}}, \tilde{x}_{i_{k}}^{\prime}$, and the coefficient semifield is generated by the elements $u_{i_{k}}, u_{\left[\gamma_{j}\right]}$, where $1 \leq k \leq d$ and $-1 \leq j \leq d+1$. Define $\pi_{*}$ on the generators $\tilde{x}_{i_{k}}, \tilde{x}_{i_{k}}^{\prime}, u_{i_{k}}, u_{\left[\gamma_{j}\right]}$ as in the statement of the theorem, and extend it to arbitrary elements by the homomorphism property.

To show that $\pi_{*}$ is well defined, we have to check that it preserves the relations between the generators. In $\tilde{\mathcal{A}}$ these relations are the exchange relations (5)

$$
\tilde{x}_{i_{k}} \tilde{x}_{i_{k}}^{\prime}=\left(\tilde{y}_{i_{k}} \prod_{i_{j} \rightarrow i_{k} \in \tilde{Q}_{\tilde{T}(\gamma)}} \tilde{x}_{i_{j}}+\prod_{i_{j} \leftarrow i_{k} \in \tilde{Q}_{\tilde{T}(\gamma)}} \tilde{x}_{i_{j}}\right) /\left(1 \oplus \tilde{y}_{i_{k}}\right),
$$

where

$$
\begin{aligned}
\tilde{y}_{i_{k}} & =u_{i_{k}} \prod_{\left[\gamma_{j}\right] \rightarrow i_{k} \in \tilde{Q}_{\tilde{T}(\gamma)}} u_{\left[\gamma_{j}\right]} \prod_{\left[\gamma_{j}\right] \leftarrow i_{k} \in \tilde{Q}_{\tilde{T}(\gamma)}} u_{\left[\gamma_{j}\right]}^{-1}, \quad \text { by equation (6) , and } \\
1 \oplus \tilde{y}_{i_{k}} & =\prod_{\left[\gamma_{j}\right] \leftarrow i_{k} \in \tilde{Q}_{\tilde{T}(\gamma)}} u_{\left[\gamma_{j}\right]}^{-1}, \quad \text { by definition of } \oplus .
\end{aligned}
$$




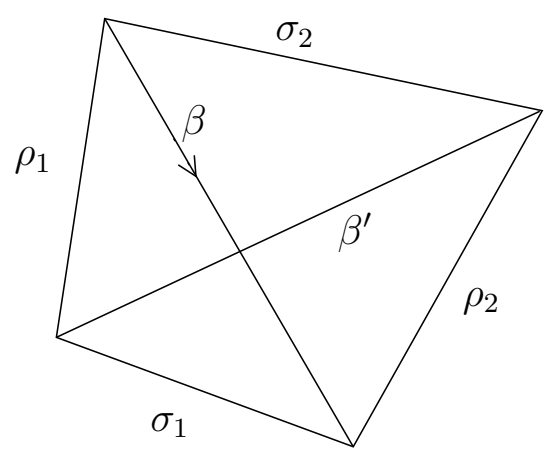

Figure 8. The quadrilateral $V$

By definition, $\pi_{*}$ maps equation (11) to

$$
x_{i_{k}} x_{i_{k}}^{\prime}=y_{i_{k}} \prod_{\left[\gamma_{j}\right] \rightarrow i_{k} \in Q_{T}} x_{\left[\gamma_{j}\right]} \prod_{i_{j} \rightarrow i_{k} \in Q_{T}} x_{i_{j}}+\prod_{\left[\gamma_{j}\right] \leftarrow i_{k} \in Q_{T}} x_{\left[\gamma_{j}\right]} \prod_{i_{j} \leftarrow i_{k} \in Q_{T}} x_{i_{j}},
$$

which, using equation (7) and the fact that the initial seed in $\mathcal{A}$ has principal coefficients, is easily seen to be an exchange relation in $\mathcal{A}$.

It remains to show that $\pi_{*}\left(\tilde{x}_{\tilde{\beta}}\right)=x_{\beta}$ whenever $\tilde{\beta}$ is an interior arc that is a lift of $\beta$. We prove this by induction on the minimal number of crossing points between $\beta$ and $T$. If this number is zero, then $\beta \in T$, and $\pi_{*}\left(\tilde{x}_{\tilde{\beta}}\right)=x_{\beta}$, by definition. Otherwise, let $\rho_{1}, \rho_{2}, \sigma_{1}, \sigma_{2}$, and $\beta^{\prime}$ be as in Lemma 5.6. Suppose without loss of generality that the relative position of these arcs is as in Figure 8 Then, in $\mathcal{A}$, we have the exchange relation

$$
x_{\beta}=\left(x_{\rho_{1}} x_{\rho_{2}} y_{\beta}^{+}+x_{\sigma_{1}} x_{\sigma_{2}} y_{\beta}^{-}\right) / x_{\beta^{\prime}},
$$

for some coefficients $y_{\beta}^{+}$and $y_{\beta}^{-}$.

Let $\tilde{\rho}_{1}$ and $\tilde{\sigma}_{2}$ be the unique lifts of $\rho_{1}$ and $\sigma_{2}$, respectively, that start at the same point as $\tilde{\beta}$. Let $\tilde{\sigma}_{1}$ and $\tilde{\beta}^{\prime}$ be the unique lifts of $\sigma_{1}$ and $\beta^{\prime}$, respectively, that start at the endpoint of $\tilde{\rho}_{1}$, and let $\tilde{\rho}_{2}$ be the unique lift of $\rho_{2}$ that starts at the endpoint of $\tilde{\sigma}_{2}$. Then $\tilde{\rho}_{1}, \tilde{\rho}_{2}, \tilde{\sigma}_{1}, \tilde{\sigma}_{2}$ form a quadrilateral in $\tilde{S}(\gamma)$ in which $\tilde{\beta}$ and $\tilde{\beta}^{\prime}$ are the diagonals. Consequently, in the cluster algebra $\tilde{\mathcal{A}}$, we have the exchange relation

$$
\tilde{x}_{\tilde{\beta}}=\left(\tilde{x}_{\tilde{\rho}_{1}} \tilde{x}_{\tilde{\rho}_{2}} \tilde{y}_{\tilde{\beta}}^{+}+\tilde{x}_{\tilde{\sigma}_{1}} \tilde{x}_{\tilde{\sigma}_{2}} \tilde{y}_{\tilde{\beta}}^{-}\right) / \tilde{x}_{\tilde{\beta}^{\prime}} \cdot
$$

Therefore

$$
\pi_{*}\left(\tilde{x}_{\tilde{\beta}}\right)=\left(\pi_{*}\left(\tilde{x}_{\tilde{\rho}_{1}}\right) \pi_{*}\left(\tilde{x}_{\tilde{\rho}_{2}}\right) \pi_{*}\left(\tilde{y}_{\tilde{\beta}}^{+}\right)+\pi_{*}\left(\tilde{x}_{\tilde{\sigma}_{1}}\right) \pi_{*}\left(\tilde{x}_{\tilde{\sigma}_{2}}\right) \pi_{*}\left(\tilde{y}_{\tilde{\beta}}^{-}\right)\right) / \pi_{*}\left(\tilde{x}_{\tilde{\beta}^{\prime}}\right) .
$$

In this formula $\tilde{y}_{\tilde{\beta}}^{+}$is a product $\tilde{y}_{\tilde{\beta}}^{+}=\tilde{u}_{\tilde{\beta}}^{+}\left(\prod u_{\left[\gamma_{j}\right]}\right)$, where $\tilde{u}_{\tilde{\beta}}^{+}$is a product of $\tilde{u}_{i_{k}}$ 's, whereas $\prod u_{\left[\gamma_{j}\right]}=u_{\tilde{\rho}_{1}}^{\delta_{1}} u_{\tilde{\rho}_{2}}^{\delta_{2}}$ with

$$
\delta_{i}= \begin{cases}0 & \text { if } \tilde{\rho}_{i} \text { is an interior arc in }(\tilde{S}(\gamma), \tilde{M}(\gamma)), \\ 1 & \text { if } \tilde{\rho}_{i} \text { is a boundary } \operatorname{arc} \operatorname{in}(\tilde{S}(\gamma), \tilde{M}(\gamma)) .\end{cases}
$$


Note that if $\delta_{i}=1$ then $\tilde{x}_{\tilde{\rho}_{i}}=1$. Similarly, $\tilde{y}_{\tilde{\beta}}^{-}=\tilde{u}_{\tilde{\beta}}^{-}\left(\prod u_{\left[\gamma_{j}\right]}\right)$, where $\tilde{u}_{\tilde{\beta}}^{-}$is a product of $\tilde{u}_{i_{k}}$ 's, whereas $\prod u_{\left[\gamma_{j}\right]}=u_{\tilde{\sigma}_{1}}^{\epsilon_{1}} u_{\tilde{\sigma}_{2}}^{\epsilon_{2}}$ with

$$
\epsilon_{i}= \begin{cases}0 & \text { if } \tilde{\sigma}_{i} \text { is an interior arc in }(\tilde{S}(\gamma), \tilde{M}(\gamma)), \\ 1 & \text { if } \tilde{\sigma}_{i} \text { is a boundary } \operatorname{arc} \operatorname{in}(\tilde{S}(\gamma), \tilde{M}(\gamma)) .\end{cases}
$$

Note that if $\epsilon_{i}=1$ then $\tilde{x}_{\tilde{\sigma}_{i}}=1$. By induction, equation (13) yields

$$
\pi_{*}\left(\tilde{x}_{\tilde{\beta}}\right)=\left(x_{\rho_{1}} x_{\rho_{2}} \pi_{*}\left(\tilde{u}_{\tilde{\beta}}^{+}\right)+x_{\sigma_{1}} x_{\sigma_{2}} \pi_{*}\left(\tilde{u}_{\tilde{\beta}}^{-}\right)\right) / x_{\beta^{\prime}},
$$

which, by equation (12), is equal to $x_{\beta}$ if and only if

$$
\pi_{*}\left(\tilde{u}_{\tilde{\beta}}^{+}\right)=y_{\beta}^{+} \quad \text { and } \quad \pi_{*}\left(\tilde{u}_{\tilde{\beta}}^{-}\right)=y_{\beta}^{-} .
$$

In order to show equation (14), it does not suffice to work with the triangulations alone, but one has to consider the corresponding quivers. We will also need to work in the universal cover $(\tilde{S}, \tilde{M})$ and its triangulation $\tilde{T}=\pi^{-1}(T)$ instead of the surface $(\tilde{S}(\gamma), \tilde{M}(\gamma))$ with triangulation $\tilde{T}(\gamma)$.

Let $\mu=\mu_{j_{s}} \circ \mu_{j_{s-1}} \circ \ldots \circ \mu_{2} \circ \mu_{1}$ be a sequence of flips in $(S, M)$ such that the quadrilateral $V$ given by the arcs $\rho_{1}, \rho_{2}, \sigma_{1}, \sigma_{2}$ and $\beta^{\prime}$ lies in $\mu T$. Each $\mu_{j}$ flips an arc $\tau_{j}$ of a triangulation of $(S, M)$. The fiber of $\tau_{j}$ in $(\tilde{S}, \tilde{M})$ is of the form $\left(\left(\tau_{j}\right)_{g}\right)_{g \in \Pi_{1}(S)}$. Let $\tilde{\mu}_{j}$ denote the (infinite) sequence of flips in each $\left(\tau_{j}\right)_{g}$ in the fiber of $\tau_{j}$ in $(\tilde{S}, \tilde{M})$. Then $\tilde{\mu}=\tilde{\mu}_{j_{s}} \circ \tilde{\mu}_{j_{s-1}} \circ \ldots \circ \tilde{\mu}_{2} \circ \tilde{\mu}_{1}$ is a sequence of flips in $(\tilde{S}, \tilde{M})$ such that every lift $\tilde{V}$ of the quadrilateral $V$ lies in $\tilde{\mu} \tilde{T}$.

On the other hand, in $(\tilde{S}(\gamma), \tilde{M}(\gamma))$, we have the lift $\bar{V}$ of $V$ given by the arcs $\tilde{\rho}_{1}, \tilde{\rho}_{2}, \tilde{\sigma}_{1}, \tilde{\sigma}_{2}$ and $\tilde{\beta}^{\prime}$. There exists a sequence $\bar{\mu}$ of flips in $(\tilde{S}(\gamma), \tilde{M}(\gamma))$ such that $\bar{V}$ lies in $\bar{\mu}(\tilde{T}(\gamma))$.

Since $(\tilde{S}(\gamma), \tilde{M}(\gamma))$ is a subsurface of $(\tilde{S}, \tilde{M})$, we can apply the same sequence of flips $\bar{\mu}$ to the triangulation $\tilde{T}$ of $(\tilde{S}, \tilde{M})$ and see that $\bar{V}$ lies in $\bar{\mu} \tilde{T}$. Clearly, $\bar{V}$ is a lift of $V$ in $(\tilde{S}, \tilde{M})$, and thus, $\bar{V}$ also lies in $\tilde{\mu} \tilde{T}$.

$\pi: Q_{\tilde{T}} \rightarrow Q_{T}$ is a Galois covering of quivers, by Theorem 5.2. Let $k$ denote the vertex in $\mu Q_{T}$ that corresponds to the arc $\beta^{\prime}$ and let $k_{g}$ denote the vertex in $\tilde{\mu} Q_{\tilde{T}}$ as well as in $\bar{\mu} Q_{\tilde{T}}$ that correspond to the $\operatorname{arc} \tilde{\beta}^{\prime}$ in $\bar{V}$.

For the purpose of this proof, we will use notations for the quiver $Q_{\tilde{T}(\gamma)}$ that are induced from $Q_{\tilde{T}}$ via the inclusion $\tilde{T}(\gamma) \subset \tilde{T}$. More precisely, the vertices of $Q_{\tilde{T}}$ are labeled as usual by tuples $(j, \sigma) \in\{1,2, \ldots, 2 n\} \times \Pi_{1}(S)$ corresponding to $\operatorname{arcs}$ of $\tilde{T}$, if $1 \leq j \leq n$, and to coefficients, if $n+1 \leq j \leq 2 n$; and we use the same labels for the vertices of the subquiver $Q_{\tilde{T}(\gamma)}$. Note that, by definition of $Q_{\tilde{T}(\gamma)}$, there are precisely $d$ vertices that carry a label $(n+i, \sigma)$ with $1 \leq i \leq n$, and these correspond to those $\operatorname{arcs}(i, \sigma)$ in $\tilde{T}$ that are interior arcs in the triangulation $\tilde{T}(\gamma)$ of the polygon $(\tilde{S}(\gamma), \tilde{M}(\gamma))$.

Then, by Theorem 5.2, the number of arrows $(n+i) \rightarrow k$ in $\mu Q_{T}$ is equal to the number of arrows $\left((n+i)_{h}\right)_{h \in \Pi_{1}(S)} \rightarrow k_{g}$ in $\tilde{\mu} Q_{\tilde{T}}$. This last number is also equal to the number of arrows $\left((n+i)_{h}\right)_{h \in \Pi_{1}(S)} \rightarrow k_{g}$ in $\bar{\mu} Q_{\tilde{T}}$, since two sequences of mutations leading to the same quadrilateral will produce the same coefficients at the diagonals of the quadrilateral, because seeds are determined by their clusters, by [FST, Theorem 5.6]. In $Q_{\tilde{T}}$, the vertex $(n+i)_{h}$ is adjacent only to the vertex $i_{h}$, thus if $(n+i)_{h} \rightarrow k_{g}$ is an arrow in $\bar{\mu} Q_{\tilde{T}}$ then the sequence $\bar{\mu}$ must flip the arc corresponding to the vertex $i_{h}$ at least once. By [Mo, pp. 40,41], we can conclude that if $(n+i)_{h} \rightarrow k_{g}$ is an arrow in $\bar{\mu} Q_{\tilde{T}}$, then the arc corresponding to the vertex 
$i_{h}$ crosses $\beta^{\prime}$ and hence, the arc corresponding to $i_{h}$ is an interior arc of the polygon $(\tilde{S}(\gamma), \tilde{M}(\gamma))$ and thus, $(n+i)_{h} \rightarrow k_{g}$ is also an arrow of $\bar{\mu} Q_{\tilde{T}(\gamma)}$.

Therefore, the number of arrows $(n+i) \rightarrow k$ in $\mu Q_{T}$ is equal to the number of arrows $\left((n+i)_{h}\right)_{h \in \Pi_{1}(S)} \rightarrow k_{g}$ in $\bar{\mu} Q_{\tilde{T}(\gamma)}$, and consequently, $y_{\beta}^{+}=\pi_{*}\left(\tilde{y}_{\tilde{\beta}}^{+}\right)$. The proof of $y_{\beta}^{-}=\pi_{*}\left(\tilde{y}_{\tilde{\beta}}^{-}\right)$is similar.

Lemma 5.8. The covering map $\pi$ induces a bijection $\bar{\pi}$ from the set of complete $(\tilde{T}(\gamma), \tilde{\gamma})$-paths in $\tilde{S}$ to set of complete $(T, \gamma)$-paths in $S$. which sends a path $\tilde{\alpha}=$ $\left(\tilde{\alpha}_{1}, \tilde{\alpha}_{2}, \ldots, \tilde{\alpha}_{2 d+1}\right)$ to the path $\bar{\pi}(\tilde{\alpha})=\left(\pi\left(\tilde{\alpha}_{1}\right), \pi\left(\tilde{\alpha}_{2}\right), \ldots, \pi\left(\tilde{\alpha}_{2 d+1}\right)\right)$.

Proof.

$\bar{\pi}$ is injective. Suppose $\bar{\pi}(\tilde{\alpha})=\bar{\pi}(\tilde{\beta})$. Each $\tilde{\alpha}_{i}$ (respectively $\tilde{\beta}_{i}$ ) is the unique lift of $\pi\left(\tilde{\alpha}_{i}\right)$ (respectively $\left.\pi\left(\tilde{\beta}_{i}\right)\right)$ that starts at the endpoint of $\tilde{\alpha}_{i-1}$ (respectively $\tilde{\beta}_{i-1}$ ), for $i=2,3, \ldots, d+1$. Since $\tilde{\alpha}_{1}$ and $\tilde{\beta}_{1}$ both start at $\tilde{s}$ it follows that $\tilde{\alpha}=\tilde{\beta}$ and $\bar{\pi}$ is injective.

$\bar{\pi}$ is surjective. For every complete $(T, \gamma)$-path $\alpha$ there is a lift $\tilde{\alpha}$ that starts at $\tilde{s}$. We have to show that $\tilde{\alpha}$ is a complete $(\tilde{T}(\gamma),(\tilde{\gamma}))$-path. Since the crossing points of $\tilde{\alpha}$ and $\tilde{\gamma}$ are $\tilde{p}_{1}, \ldots, \tilde{p}_{d}$ in order, $\tilde{\alpha}$ satisfies condition (T1). Condition (T2) holds since $\tilde{S}(\gamma)$ is simply connected.

5.4. Cluster expansion in $\tilde{\mathcal{A}}$. In this section, we will use Theorem 3.2 for simply connected surfaces to compute the cluster expansion of $\tilde{x}_{\tilde{\gamma}}$ in the cluster algebra $\tilde{A}$. Since $\tilde{A}$ does not have principal coefficients, we cannot use Theorem 3.2 directly.

Let $x_{\tilde{\gamma}}$ be the cluster expansion in the cluster algebra associated to $(\tilde{S}(\gamma), \tilde{M}(\gamma))$ with principal coefficient in the initial seed associated to the triangulation $\tilde{T}(\gamma)$. By Theorem 2.5, we have

$$
\tilde{x}_{\tilde{\gamma}}=\frac{\left.x_{\tilde{\gamma}}\right|_{\tilde{\mathcal{F}}}\left(\tilde{x}_{i_{1}}, \ldots, \tilde{x}_{i_{d}} ; \tilde{y}_{i_{1}}, \ldots, \tilde{y}_{i_{d}}\right)}{x_{\tilde{\gamma}} \mid \tilde{\mathbb{P}}\left(1,1, \ldots, 1, \tilde{y}_{i_{1}}, \ldots, \tilde{y}_{i_{d}}\right)} .
$$

Using Theorem 3.2, we get

$$
\tilde{x}_{\tilde{\gamma}}=\frac{\left.\left.\sum_{\tilde{\alpha}} x(\tilde{\alpha})\right|_{\tilde{\mathcal{F}}}\left(\tilde{x}_{i_{1}}, \ldots, \tilde{x}_{i_{d}}\right) y(\tilde{\alpha})\right|_{\tilde{\mathcal{F}}}\left(\tilde{y}_{i_{1}}, \ldots, \tilde{y}_{i_{d}}\right)}{\left.\sum_{\tilde{\alpha}} y(\tilde{\alpha})\right|_{\tilde{\mathbb{P}}}\left(\tilde{y}_{i_{1}}, \ldots, \tilde{y}_{i_{d}}\right)},
$$

where both sums are over all complete $(\tilde{T}(\gamma), \tilde{\gamma})$-paths $\tilde{\alpha}$. We compute the right hand side of equation (15) in the following Lemma.

Lemma 5.9. Let $\tilde{\alpha}$ be a complete $(\tilde{T}(\gamma), \tilde{\gamma})$-path. Then

$$
x(\tilde{\alpha}))\left.\right|_{\tilde{\mathcal{F}}}\left(\tilde{x}_{i_{1}}, \ldots, \tilde{x}_{i_{d}}\right)=\frac{\prod_{k} \tilde{x}_{\tilde{\alpha}_{k}}}{\tilde{x}_{i_{1}} \cdots \tilde{x}_{i_{d}}}
$$

where the product is over all odd integers $k$ such that $\tilde{\alpha}_{k}$ is an interior arc in $(\tilde{S}(\gamma), \tilde{M}(\gamma))$;

$$
\left.y(\tilde{\alpha})\right|_{\tilde{\mathcal{F}}}\left(\tilde{y}_{i_{1}}, \ldots, \tilde{y}_{i_{d}}\right)=\left.\sum_{\tilde{\beta}} y(\tilde{\beta})\right|_{\tilde{\mathbb{P}}}\left(\tilde{y}_{i_{1}}, \ldots, \tilde{y}_{i_{d}}\right)\left(\prod_{\ell} u_{i_{\ell}}\right)\left(\prod_{k} u_{\tilde{\alpha}_{k}}\right)
$$

where the sum is over all complete $(\tilde{T}(\gamma), \tilde{\gamma})$-paths $\tilde{\beta}$, the first product is over all integers $\ell$ such that $\tilde{\alpha}_{2 \ell}$ is $\tilde{\gamma}$-oriented, and the second product is over all odd integers $k$ such that $\tilde{\alpha}_{k}$ is a boundary arc in $(\tilde{S}(\gamma), \tilde{M}(\gamma))$. 
Proof. Evaluating $x(\tilde{\alpha})$ in $\tilde{x}_{i_{1}}, \ldots, \tilde{x}_{i_{d}}$ sets all the terms $\tilde{x}_{\tilde{\alpha}_{k}}$ with $\tilde{\alpha}_{k}$ a boundary arc in $(\tilde{S}(\gamma), \tilde{M}(\gamma))$ equal to 1 . Thus formula (16) follows from the fact that all even $\operatorname{arcs} \tilde{\alpha}_{2 k}$ of $\tilde{\alpha}$ are interior arcs in $(\tilde{S}(\gamma), \tilde{M}(\gamma))$.

Formula (17) is a consequence of the following three claims.

Claim 1: For $k=0,1, \ldots, d$, the exponent of $u_{\left[\gamma_{k}\right]}$ in $\left.y(\tilde{\alpha})\right|_{\tilde{\mathcal{F}}}\left(\tilde{y}_{i_{1}}, \ldots, \tilde{y}_{i_{d}}\right)$ is 1 if and only if $\tilde{\alpha}_{2 k+1}=\tilde{\tau}_{\left[\gamma_{k}\right]}$.

Proof of Claim 1. By definition,

$$
\left.y(\tilde{\alpha})\right|_{\tilde{\mathcal{F}}}\left(\tilde{y}_{i_{1}}, \ldots, \tilde{y}_{i_{d}}\right)=\prod_{k: \tilde{\alpha}_{2 k}} \tilde{y}_{i_{k}},
$$

and if $2 \leq k \leq d-1$ then

$$
\tilde{y}_{i_{k}}=u_{i_{k}} u_{\left[\gamma_{k}\right]}^{\delta_{k}} u_{\left[\gamma_{k-1}\right]}^{\delta_{k-1}}, \text { with }
$$

$\delta_{j}= \begin{cases}1 & \text { if } \tilde{\tau}_{\left[\gamma_{j}\right]} \text { follows } \tilde{\tau}_{i_{k}} \text { in the clockwise orientation in } \tilde{\Delta}_{j} \\ -1 & \text { if } \tilde{\tau}_{\left[\gamma_{j}\right]} \text { follows } \tilde{\tau}_{i_{k}} \text { in the counter-clockwise orientation in } \tilde{\Delta}_{j},\end{cases}$ for $j=k-1, k$. Also,

$$
\tilde{y}_{i_{1}}=u_{i_{1}} u_{\left[\gamma_{0}\right]} u_{\left[\gamma_{-1}\right]}^{-1} \text {, and } \tilde{y}_{i_{d}}=u_{i_{d}} u_{\left[\gamma_{d}\right]} u_{\left[\gamma_{d+1}\right]}^{-1} .
$$

Therefore, the exponent of $u_{\left[\gamma_{0}\right]}$ is 1 if and only if $\tilde{\alpha}_{2}$ is $\tilde{\gamma}$-oriented, which is the case if and only if $\tilde{\alpha}_{1}=\tilde{\tau}_{\left[\gamma_{0}\right]}$. This proves the case $k=0$. The exponent of $u_{\left[\gamma_{d}\right]}$ is 1 if and only if $\tilde{\alpha}_{2 d}$ is $\tilde{\gamma}$-oriented, which is the case if and only if $\tilde{\alpha}_{2 d+1}=\tilde{\tau}_{\left[\gamma_{d}\right]}$. This proves the case $k=d$.

Now suppose that $0<k<d$. The exponent of $u_{\left[\gamma_{k}\right]}$ is 1 if and only if,

- either $\tilde{\alpha}_{2 k+2}\left(=\tilde{\tau}_{i_{k+1}}\right)$ is $\tilde{\gamma}$-oriented, $\tilde{\alpha}_{2 k}\left(=\tilde{\tau}_{i_{k}}\right)$ is not $\tilde{\gamma}$-oriented, and the sequence

$$
\tilde{\tau}_{i_{k}}, \tilde{\tau}\left[\gamma_{k}\right], \tilde{\tau}_{i_{k}+1}
$$

corresponds to the counter-clockwise orientation of the triangle $\tilde{\Delta}_{k}$,

- or $\tilde{\alpha}_{2 k}\left(=\tilde{\tau}_{i_{k}}\right)$ is $\tilde{\gamma}$-oriented, $\tilde{\alpha}_{2 k+2}\left(=\tilde{\tau}_{i_{k+1}}\right)$ is not $\tilde{\gamma}$-oriented, and the sequence

$$
\tilde{\tau}_{i_{k}}, \tilde{\tau}\left[\gamma_{k}\right], \tilde{\tau}_{i_{k+1}}
$$

corresponds to the clockwise orientation of the triangle $\tilde{\Delta}_{k}$.

In both cases, we have $\tilde{\alpha}_{2 k+1}=\tilde{\tau}_{\left[\gamma_{k}\right]}$, see Figure 9 .

Conversely, if $\tilde{\alpha}_{2 k+1}=\tilde{\tau}_{\left[\gamma_{k}\right]}$, then the segment

$$
\left(\tilde{\alpha}_{2 k}, \tilde{\alpha}_{2 k+1}, \tilde{\alpha}_{2 k+2}\right)=\left(\tilde{\tau}_{i_{k}}, \tilde{\tau}_{\left[\gamma_{k}\right]}, \tilde{\tau}_{i_{k+1}}\right)
$$

goes around the boundary of $\tilde{\Delta}_{k}$ exactly once. If this segment corresponds to the clockwise orientation of $\tilde{\Delta}_{k}$ then $\tilde{\alpha}_{2 k}$ is $\tilde{\gamma}$-oriented and $\tilde{\alpha}_{2 k+2}$ is not, and the exponent of $u_{\left[\gamma_{k}\right]}$ is 1 ; and if it corresponds to the counterclockwise orientation of $\tilde{\Delta}_{k}$ then $\tilde{\alpha}_{2 k+2}$ is $\tilde{\gamma}$-oriented and $\tilde{\alpha}_{2 k}$ is not, and, again,the exponent of $u_{\left[\gamma_{k}\right]}$ is 1 . This shows Claim 1.

Claim 2: The exponent of $u_{\left[\gamma_{k}\right]}$ in $\left.\tilde{y}(\tilde{\alpha})\right|_{\tilde{\mathcal{F}}}\left(\tilde{y}_{i_{1}}, \ldots, \tilde{y}_{i_{d}}\right)$ is -1 if and only if

- either $k=-1$ and $\tilde{\alpha}_{1} \neq \tilde{\tau}_{\left[\gamma_{-1}\right]}$,

- or $k=d+1$ and $\tilde{\alpha}_{2 d+1} \neq \tilde{\tau}_{\left[\gamma_{d+1}\right]}$.

Proof of Claim 2. If $\tilde{\alpha}_{1} \neq \tilde{\tau}_{\left[\gamma_{-1}\right]}$ then $\tilde{\alpha}_{1}=\tilde{\tau}_{\left[\gamma_{0}\right]}$, thus $\tilde{\alpha}_{2}$ is $\tilde{\gamma}$-oriented and the exponent of $u_{\left[\gamma_{-1}\right]}$ is -1 . Similarly, if $\tilde{\alpha}_{2 d+1} \neq \tilde{\tau}_{\left[\gamma_{d+1}\right]}$ then $\tilde{\alpha}_{2 d+1}=\tilde{\tau}_{\left[\gamma_{d}\right]}$, thus $\tilde{\alpha}_{2 d}$ is $\tilde{\gamma}$-oriented and the exponent of $u_{\left[\gamma_{d+1}\right]}$ is -1 .

Let us suppose now that $k \neq-1, d+1$ and $u_{\left[\gamma_{k}\right]}$ has exponent equal to -1 . Then 

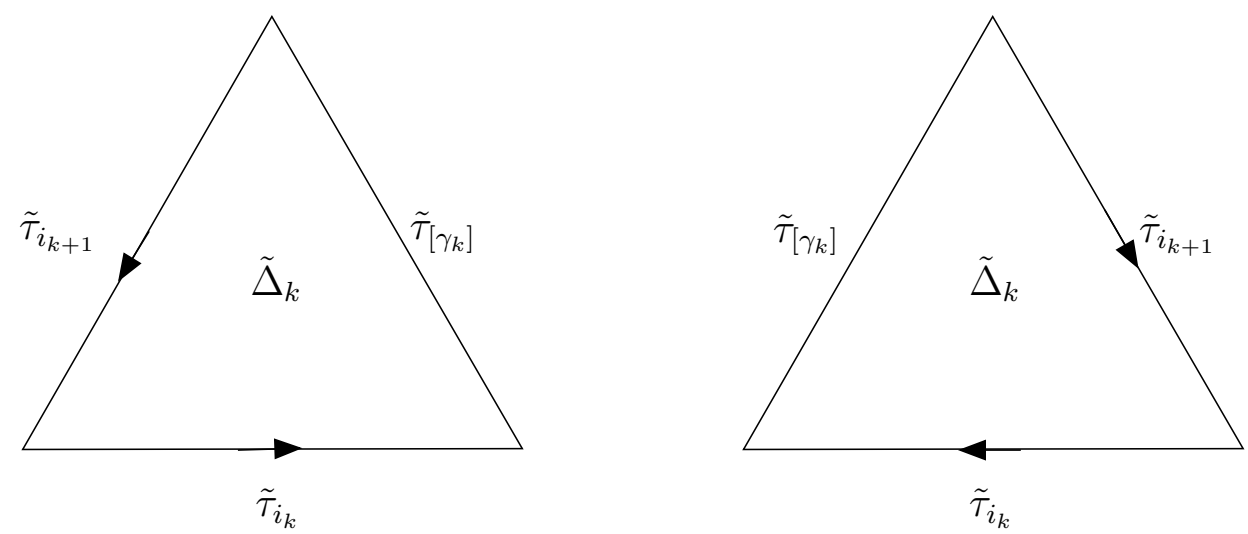

Figure 9. The triangle $\tilde{\Delta}_{k}$. On the left, $\tilde{\tau}_{i_{k+1}}$ is $\tilde{\gamma}$-oriented and $\tilde{\tau}_{i_{k}}$ is not; on the right, $\tilde{\tau}_{i_{k}}$ is $\tilde{\gamma}$-oriented and $\tilde{\tau}_{i_{k+1}}$ is not

- either $\tilde{\alpha}_{2 k}$ is $\tilde{\gamma}$-oriented, $\tilde{\alpha}_{2 k+2}$ is not and the sequence $\tilde{\tau}_{i_{k}}, \tilde{\tau}_{\left[\gamma_{k}\right]}, \tilde{\tau}_{i_{k+1}}$ corresponds to the counter-clockwise orientation of the triangle $\tilde{\Delta}_{k}$,

- or $\tilde{\alpha}_{2 k+2}$ is $\tilde{\gamma}$-oriented, $\tilde{\alpha}_{2 k}$ is not and the sequence $\tilde{\tau}_{i_{k}}, \tilde{\tau}_{\left[\gamma_{k}\right]}, \tilde{\tau}_{i_{k+1}}$ corresponds to the clockwise orientation of the triangle $\tilde{\Delta}_{k}$.

Both cases are impossible. Indeed, in the first case, we would have

$$
\left(\tilde{\alpha}_{2 k}, \tilde{\alpha}_{2 k+1}, \tilde{\alpha}_{2 k+2}\right)=\left(\tilde{\tau}_{i_{k}}, \tilde{\tau}_{i_{k+1}}, \tilde{\tau}_{i_{k+1}}\right) \text {, }
$$

contradicting the assumption that $\tilde{\alpha}_{2 k}$ is $\tilde{\gamma}$-oriented and $\tilde{\alpha}_{2 k+2}$ is not; and in the second case we would have

$$
\left(\tilde{\alpha}_{2 k}, \tilde{\alpha}_{2 k+1}, \tilde{\alpha}_{2 k+2}\right)=\left(\tilde{\tau}_{i_{k}}, \tilde{\tau}_{i_{k+1}}, \tilde{\tau}_{i_{k+1}}\right)
$$

contradicting the assumption that $\tilde{\alpha}_{2 k+2}$ is $\tilde{\gamma}$-oriented and $\tilde{\alpha}_{2 k}$ is not. This shows that we cannot have $k \neq-1, d+1$, and claim 2 follows.

Claim 3: $\left.\sum_{\tilde{\beta}} \tilde{y}(\tilde{\beta})\right|_{\tilde{\mathbb{P}}}\left(\tilde{y}_{i_{1}}, \ldots, \tilde{y}_{i_{d}}\right)=u_{\left[\gamma_{-1}\right]}^{-1} u_{\left[\gamma_{d+1}\right]}^{-1}$.

Proof of Claim 3. This follows from the definition of the addition $\oplus$ in $\tilde{\mathbb{P}}$, together with Claim 2 and the fact that there always exist complete $(\tilde{T}(\gamma), \tilde{\gamma})$-paths $\tilde{\alpha}$ and $\tilde{\beta}$ such that $\tilde{\alpha}_{1}=\tilde{\tau}_{\left[\gamma_{-1}\right]}$ and $\tilde{\beta}_{2 d+1}=\tilde{\tau}_{\left[\gamma_{d+1}\right]}$.

Applying Lemma 5.9 to equation (15), we get

Proposition 5.10. Let $\tilde{x}_{\tilde{\gamma}}$ be the cluster variable in $\tilde{A}$ corresponding an arc $\tilde{\gamma}$ in $(\tilde{S}(\gamma), \tilde{M}(\gamma))$. Then the cluster expansion of $\tilde{x}$ in the initial seed associated to the triangulation $\tilde{T}(\gamma)$ is given by

$$
\tilde{x}_{\tilde{\gamma}}=\sum_{\tilde{\alpha}} \frac{\prod_{j} \tilde{x}_{\tilde{\alpha}_{j}} \prod_{k} u_{\tilde{\alpha}_{k}} \prod_{\ell} u_{i_{\ell}}}{\tilde{x}_{i_{1}}, \ldots, \tilde{x}_{i_{d}}},
$$

where the sum is over all complete $(\tilde{T}(\gamma), \tilde{\gamma})$-paths $\tilde{\alpha}$, the first product is over all odd integers $j$ such that $\tilde{\alpha}_{j}$ is an interior arc in $(\tilde{S}(\gamma), \tilde{M}(\gamma))$, the second product is over all odd integers $k$ such that $\tilde{\alpha}_{k}$ is a boundary arc in $(\tilde{S}(\gamma), \tilde{M}(\gamma))$, and the third product is over all integers $\ell$ such that $\tilde{\alpha}_{2 \ell}$ is $\tilde{\gamma}$-oriented. 
5.5. Proof of Theorem 3.2 in the general case. In this subsection, we will prove Theorem 3.2 for arbitrary unpunctured surfaces $S$.

Let $\gamma$ be any arc in $(S, M)$, let $\tilde{\gamma}$ be a lift of $\gamma$ in $(\tilde{S}, \tilde{M})$, and let $(\tilde{S}(\gamma), \tilde{M}(\gamma))$ be the simply connected surface constructed in section [5.3. Let $\tilde{A}$ be the cluster algebra with combined boundary and principal coefficients introduced in Definition 4. By Theorem [5.7 we have $x_{\gamma}=\pi_{*}\left(\tilde{x}_{\tilde{\gamma}}\right)$, and from Proposition 5.10 we get

$$
x_{\gamma}=\sum_{\tilde{\alpha}} \frac{\prod_{j} \pi_{*}\left(\tilde{x}_{\tilde{\alpha}_{j}}\right) \prod_{k} \pi_{*}\left(u_{\tilde{\alpha}_{k}}\right) \prod_{\ell} \pi_{*}\left(u_{i_{\ell}}\right)}{\pi_{*}\left(\tilde{x}_{i_{1}}, \ldots, \tilde{x}_{i_{d}}\right)},
$$

where the sum is over all complete $(\tilde{T}(\gamma), \tilde{\gamma})$-paths $\tilde{\alpha}$, the first product is over all odd integers $j$ such that $\tilde{\alpha}_{j}$ is an interior arc in $(\tilde{S}(\gamma), \tilde{M}(\gamma))$, the second product is over all odd integers $k$ such that $\tilde{\alpha}_{k}$ is a boundary arc in $(\tilde{S}(\gamma), \tilde{M}(\gamma))$, and the third product is over all integers $\ell$ such that $\tilde{\alpha}_{2 \ell}$ is $\tilde{\gamma}$-oriented. By Theorem 5.7 Lemma 5.8 and Lemma 5.4, it follows that

$$
x_{\gamma}=\sum_{\alpha} \frac{\prod_{j} x_{\alpha_{j}}}{x_{i_{1}}, \ldots, x_{i_{d}}} \prod_{\ell} y_{i_{\ell}}
$$

where the sum is over all complete $(T, \gamma)$-paths $\alpha$, the first product is over all odd integers $j$ and the second product is over all integers $\ell$ such that $\alpha_{2 \ell}$ is $\gamma$-oriented. Thus

$$
x_{\gamma}=\sum_{\alpha} x(\alpha) y(\alpha)
$$

where $\alpha$ runs over all complete $(T, \gamma)$-paths in $(S, M)$, as required.

\section{F-POLYNOMIALS AND $g$-VECTORS}

In this section we study the $F$-polynomials and the $g$-vectors introduced in FZ2.

6.1. F-polynomials. By definition, the $F$-polynomial $F_{\gamma}$ is obtained from the Laurent polynomial $x_{\gamma}$ (with principal coefficients) by substituting the cluster variables $x_{1}, x_{2}, \ldots, x_{n}$ by 1 .

Theorem 6.1. Let $T$ be a triangulation of an unpunctured surface $(S, M)$, and let $\gamma$ be an arc. Then the F-polynomial of $\gamma$ is given by

$$
F_{\gamma}=\sum_{\alpha} y(\alpha)
$$

where the sum is over all complete $(T, \gamma)$-paths in $(S, M)$.

Proof. This follows immediately from Theorem 3.2 .

Theorem 6.2. [FZ2, Conjecture 5.4] Let $T$ be a triangulation of an unpunctured surface $(S, M)$, and let $\gamma$ be an arc. Then the F-polynomial of $\gamma$ has constant term 1.

Proof. We have to show that for any arc $\gamma$, there is precisely one complete $(T, \gamma)$ path $\alpha^{0}$ such that none of its even $\operatorname{arcs} \alpha_{2 k}$ is $\gamma$-oriented. As usual, let $d$ denote the number of crossings between $\gamma$ and $T$. If $d=0$ then $\gamma \in T$ and there is nothing to prove. So suppose that $d \geq 1$ and let $p_{1}, p_{2}, \ldots, p_{d}$ be the crossing points of $\gamma$ and $T$ in order of occurrence on $\gamma$, and let $\tau_{i_{j}} \in T$ be such that $p_{j}$ lies on $\tau_{i_{j}}$. Let $s$ be the starting point of $\gamma$ and let $t$ be its endpoint. For $k=1,2, \ldots, d$, label the 
endpoints of $\tau_{i_{k}}$ by $s_{k}, t_{k}$ in such a way that going along $\tau_{i_{k}}$ from $s_{k}$ to $t_{k}$ is not $\gamma$-oriented. Note that $s_{k}$ can be equal to $t_{k}$. We have either $s_{k}=s_{k+1}$ or $t_{k}=t_{k+1}$. Also note that $i_{k}=i_{k^{\prime}}$ does not necessarily imply that $s_{k}=s_{k^{\prime}}$ since $\gamma$ may cross $\tau_{i_{k}}$ several times and in both directions. Now define $\alpha$ as follows. Let $\alpha_{1}=\tau_{\left[\gamma_{-1}\right]}$ the unique arc in $T$ that is a side of the triangle $\Delta_{0}$ and goes from $s$ to $s_{1}$. For $k=1,2, \ldots, d-1$, let $\alpha_{2 k}$ be the arc $\tau_{i_{k}}$ running in the non $\gamma$-oriented direction, and let $\alpha_{2 k+1}$ be the arc $\tau_{i_{k}}$ running in the $\gamma$-oriented direction if $s_{k+1}=s_{k}$, and let $\alpha_{2 k+1}$ be the arc $\tau_{i_{k+1}}$ running in the $\gamma$-oriented direction if $s_{k+1} \neq s_{k}$. Finally, let $\alpha_{2 d}=\tau_{i_{d}}$ running in the non $\gamma$-oriented direction, and let $\alpha_{2 d+1}=\tau_{\gamma_{\left[\gamma_{d+1}\right]}}$ the unique arc in in $T$ that is a side of the triangle $\Delta_{d}$ and goes from $t_{d}$ to $t$.

Then $\alpha$ is a complete $(T, \gamma)$-path and none of its even arcs is $\gamma$-oriented.

To show uniqueness, it suffices to observe that specifying the even arcs and their orientation, together with the homotopy condition (T2), completely determines the odd arcs and hence $\alpha$.

Example 6.3. In Example 3.4 the path $\alpha^{0}$ is the path

$$
\left(\tau_{7}, \tau_{1}, \tau_{1}, \tau_{3}, \tau_{5}, \tau_{5}, \tau_{11}\right)
$$

and in Example 3.5 the path $\alpha^{0}$ is the path

$$
\left(\tau_{5}, \tau_{1}, \tau_{1}, \tau_{2}, \tau_{3}, \tau_{3}, \tau_{3}, \tau_{4}, \tau_{1}, \tau_{1}, \tau_{8}\right)
$$

Corollary 6.4. [FZ2, Conjecture 5.5] Each F-polynomial has a unique monomial of maximal degree. Furthermore this monomial has coefficient 1 and is divisible by all the other occurring monomials.

Proof. This follows from Theorem 6.2 and [FZ2, Proposition 5.3]

6.2. $g$-vectors. We keep the notation of section 6.1 Let $\tilde{B}_{T}$ be the $2 n \times n$ matrix associated to the triangulation $T$ as in section 2.4 and let $B_{T}$ be the upper $n \times n$ part of $\tilde{B}_{T}$. It has been shown in FZ2 that, for any cluster variable $x_{\gamma}$ in $\mathcal{A}$, its Laurent expansion in the initial seed $\left(\mathbf{x}_{T}, \mathbf{y}_{T}, B_{T}\right)$ is homogeneous with respect to the grading given by $\operatorname{deg}\left(x_{i}\right)=\mathbf{e}_{i}$ and $\operatorname{deg}\left(y_{i}\right)=B_{T} \mathbf{e}_{i}$, where $\mathbf{e}_{i}=(0, \ldots, 0,1,0, \ldots, 0) \in \mathbb{Z}^{n}$ with 1 at position $i$. By definition, the $g$-vector $g_{\gamma}$ of a cluster variable $x_{\gamma}$ is the degree of its Laurent expansion with respect to this grading.

Because of Theorem 6.2 we also have $g_{\gamma}=\operatorname{deg}\left(x\left(\alpha^{0}\right)\right)$, where $\alpha^{0}$ is the unique $(T, \gamma)$-path with $y\left(\alpha^{0}\right)=1$. As in the proof of Theorem 6.2, let $s_{k}$ be the startpoint of $\tau_{i_{k}}$ in the non- $\gamma$-oriented direction, $k=1,2, \ldots, d$. Let

$$
\begin{aligned}
& I^{-}=\left\{i_{k} \mid k \in[1, d] \text { and } s_{k-1}=s_{k} \neq s_{k+1}\right\} \\
& I^{+}=\left\{i_{k} \mid k \in[2, d-1] \text { and } s_{k-1} \neq s_{k}=s_{k+1}\right\} \cup\left\{\left[\gamma_{-1}\right],\left[\gamma_{d+1}\right]\right\}
\end{aligned}
$$

where we use the convention that $s_{0}=s_{1}$ and $s_{d} \neq s_{d+1}$, so that $I^{-}$is well defined. Then we have

$$
x\left(\alpha^{0}\right)=\prod_{h \in I^{+}} x_{h} \prod_{h \in I^{-}} x_{h}^{-1} ;
$$

recall that $x_{h}=1$ if $\tau_{h}$ is a boundary arc.

Define $\mathbf{e}_{1}, \mathbf{e}_{2}, \ldots, \mathbf{e}_{n}$ to be the standard basis vectors of $\mathbb{Z}^{n}$, and let $\mathbf{e}_{h}=$ $(0, \ldots, 0)$ if $\tau_{h}$ is a boundary arc. We have proved the following theorem: 
Theorem 6.5. Let $T$ be a triangulation of an unpunctured surface $(S, M)$, and let $\gamma$ be an arc. Then the g-vector of $\gamma$ is given by

$$
g_{\gamma}=\sum_{h \in I^{+}} \mathbf{e}_{h}-\sum_{h \in I^{-}} \mathbf{e}_{h}
$$

Example 6.6. In Example 3.4, we have

$$
I^{-}=\{3\} \quad I^{+}=\{7,12\} \quad g_{\gamma}=(0,0,-1,0,0),
$$

and in Example 3.5, we have

$$
I^{-}=\{2,4\} \quad I^{+}=\{3,5,8\} \quad g_{\gamma}=(0,-1,1,-1) .
$$

Let us remark also the following useful fact.

Lemma 6.7. Let $x_{\gamma}=\sum_{\alpha} x(\alpha) y(\alpha)$ be the cluster expansion of Theorem 3.2 of a cluster variable $x_{\gamma}$, and let $\alpha, \beta$ be two complete $(T, \gamma)$-paths. Then

$$
x(\alpha) y(\alpha)=x(\beta) y(\beta) \Leftrightarrow y(\alpha)=y(\beta)
$$

Proof. Suppose $y(\alpha)=y(\beta)$. Because of the homogeneity of the expansion proved in FZ2, we have $\operatorname{deg}(x(\alpha))=\operatorname{deg}(x(\beta))$, and thus $x(\alpha)=x(\beta)$, since the degree of the monomial $x(\alpha)$ determines $x(\alpha)$ uniquely. Thus $x(\alpha) y(\alpha)=$ $x(\beta) y(\beta)$. The other implication is trivial.

\section{Arbitrary coefficients}

Let $(S, M)$ be an unpunctured surface and $T$ a triangulation of $(S, M)$. Denote by $\mathcal{A}=\mathcal{A}\left(\mathbf{x}, \mathbf{y}, B_{T}\right)$ be the cluster algebra with principal coefficients in the initial seed $\left(\mathbf{x}, \mathbf{y}, B_{T}\right)$.

Let $(\hat{\mathbb{P}}, \oplus, \cdot)$ be an arbitrary semifield, let $\hat{\mathcal{A}}$ be a cluster algebra over the ground ring $\mathbb{Z} \hat{\mathbb{P}}$ with an arbitrary coefficient system and initial seed $\left(\mathbf{x}, \hat{\mathbf{y}}, B_{T}\right)$, where $\hat{\mathbf{y}}=$ $\left\{\hat{y}_{1}, \ldots, \hat{y}_{n}\right\}$ is an initial coefficient vector, $\hat{y}_{i} \in \hat{\mathbb{P}}$.

Let $\gamma$ be an arc and $x_{\gamma}$ and $\hat{x}_{\gamma}$ the corresponding cluster variables in $\mathcal{A}$ and $\hat{\mathcal{A}}$ respectively. Denote by $\hat{\mathcal{F}}$ the field of rational functions $\mathbb{Q} \hat{\mathbb{P}}\left(x_{1}, \ldots, x_{n}\right)$. Then $\hat{\mathcal{F}}$ is the ambient field of the cluster algebra $\hat{\mathcal{A}}$.

By Theorem 2.5, we have

$$
\hat{x}_{\gamma}=\frac{\left.x_{\gamma}\right|_{\hat{\mathcal{F}}}\left(x_{1}, \ldots, x_{n} ; \hat{y}_{1}, \ldots, \hat{y}_{n}\right)}{\left.F_{\gamma}\right|_{\hat{\mathbb{P}}}\left(\hat{y}_{1}, \ldots, \hat{y}_{n}\right)},
$$

where $\left.x_{\gamma}\right|_{\hat{\mathcal{F}}}\left(x_{1}, \ldots, x_{n} ; \hat{y}_{1}, \ldots, \hat{y}_{n}\right)$ denotes the evaluation of the Laurent polynomial $x_{\gamma}$ in $\left(x_{1}, \ldots, x_{n} ; \hat{y}_{1}, \ldots, \hat{y}_{n}\right)$ and $\left.F_{\gamma}\right|_{\hat{p}}\left(\hat{y}_{1}, \ldots, \hat{y}_{n}\right)$ denotes the evaluation of the $F$ polynomial $F_{\gamma}$ in $\hat{y}_{1}, \ldots, \hat{y}_{n}$ in the semifield $\hat{\mathbb{P}}$.

Using Theorem 3.2, we get

$$
\hat{x}_{\gamma}=\frac{\sum_{\alpha} x(\alpha) \hat{y}(\alpha)}{\left.\sum_{\alpha} \hat{y}(\alpha)\right|_{\hat{\mathbb{P}}}}
$$

where $\hat{y}(\alpha)=y(\alpha)$ evaluated in $\hat{y}_{1}, \ldots, \hat{y}_{n}$; and $\left.\hat{y}(\alpha)\right|_{\hat{\mathbb{P}}}$ is $\hat{y}(\alpha)$ evaluated in $\hat{\mathbb{P}}$, that is the addition + is replaced by the addition $\oplus$.

If $\hat{\mathcal{A}}$ is of geometric type, i.e. $\hat{\mathbb{P}}=\operatorname{Trop}\left(u_{1}, \ldots, u_{\ell}\right)$ is a tropical semifield, then $\left.F_{\gamma}\right|_{\hat{\mathbb{P}}}\left(\hat{y}_{1}, \ldots, \hat{y}_{n}\right)$ is equal to $\prod_{i=1}^{\ell} u_{i}^{d(i)}$, where $d(i)$ is the minimum of all exponents of $u_{i} F_{\gamma} \mid\left(\hat{y}_{1}, \ldots, \hat{y}_{n}\right)$. By Theorem [6.2] $F_{\gamma}$ has constant term 1 , and thus $d(i) \leq 0$. 
In particular, $\left.F_{\gamma}\right|_{\hat{\mathbb{P}}}\left(\hat{y}_{1}, \ldots, \hat{y}_{n}\right)$ is a monomial, and then Corollary 3.6 implies that the right hand side of equation (20) is a polynomial in $x_{1}, \ldots, x_{n}$ with coefficients in $\mathbb{Z}_{\geq 0} \hat{\mathbb{P}}$. We have shown the following theorem known as the positivity conjecture.

Theorem 7.1. Let $(S, M)$ be an unpunctured surface and let $\hat{\mathcal{A}}=\hat{\mathcal{A}}(\mathbf{x}, \hat{\mathbf{y}}, B)$ be a cluster algebra of geometric type associated to some triangulation of $(S, M)$. Let $u$ be any cluster variable and let

$$
u=\frac{f\left(x_{1}, \ldots, x_{n}\right)}{x_{1}^{d_{1}} \ldots x_{n}^{d_{n}}}
$$

be the expansion of $u$ in the cluster $\mathbf{x}=\left\{x_{1}, \ldots, x_{n}\right\}$, where $f \in \mathbb{Z} \hat{\mathbb{P}}\left[x_{1}, \ldots, x_{n}\right]$. Then the coefficients of $f$ are non-negative integer linear combinations of elements in $\hat{\mathbb{P}}$.

\section{Further APPLiCATIONS}

8.1. Euler Poincaré Characteristics. In this section, we compare our cluster expansion formula to a formula obtained recently by Fu and Keller [FK based on earlier formulas in $\mathrm{CC}, \mathrm{CK}, \mathrm{CK} 2, \mathrm{~Pa}$. Their formula is valid for cluster algebras that admit a categorification by a 2-Calabi-Yau category, and, combining results of [A] and $\mathrm{ABCP}, \mathrm{LF}$, such a categorification exists in the case of cluster algebras associated to unpunctured surfaces.

Particularly nice are the cases where the surface is a disc or an annulus. In these cases the 2-Calabi-Yau category is the cluster category of type $A_{m-3}$, if the surface is a disc with $m$ marked points, and of type $\tilde{A}_{p, q}$, if the surface is an annulus with $p$ marked points on one boundary component and $q$ marked points on the other. Comparing the cluster expansions in these cases we shall obtain a formula for the Euler-Poincaré characteristics of certain Grassmannian varieties.

We recall some facts from cluster-tilting theory, see BMRRT, BMR1, CCS, ABS, CK2 for details. Let $(S, M)$ be an unpunctured surface such that $S$ is either a disc or an annulus. As usual, denote by $n$ the number of interior arcs in any triangulation of $(S, M)$. If $S$ is an annulus, let $p$ be the number of marked points on one boundary component and $q$ the number of marked points on the other. Define

$$
Q=\left\{\begin{array}{l}
1 \longrightarrow 2 \longrightarrow \cdots \rightarrow n-1 \longrightarrow n \\
\longrightarrow 2 \longrightarrow \cdots \rightarrow p \rightarrow p+1 \\
\longrightarrow p+2 \rightarrow \cdots \rightarrow p+q
\end{array}\right.
$$

Let $A$ be the path algebra of $Q$ over an algebraically closed field and let $\mathcal{C}$ be its cluster category.

The indecomposable rigid objects of $\mathcal{C}$ are in bijection with the cluster variables of the cluster algebra $\mathcal{A}$ associated to the surface $(S, M)$, hence, in bijection with the arcs in $(S, M)$. Denote $X_{\gamma}$ the indecomposable rigid object of $\mathcal{C}$ that corresponds to the arc $\gamma$. The clusters in $\mathcal{A}$, and hence the triangulations of $(S, M)$, are in bijection with the cluster-tilting objects in $\mathcal{C}$. Denote by $T_{\mathcal{C}}=$ $\left\{X_{\tau_{1}}, X_{\tau_{2}}, \ldots, X_{\tau_{n}}\right\}$ the cluster- tilting object corresponding to the triangulation $T=\left\{\tau_{1}, \tau_{2}, \ldots, \tau_{n}, \tau_{n+1}, \ldots, \tau_{n+m}\right\}$. The endomorphism algebra $\operatorname{End}_{\mathcal{C}}\left(T_{\mathcal{C}}\right)$ of this cluster-tilting object is called cluster-tilted algebra. It has been shown in BMR1 
that $\operatorname{Hom}_{\mathcal{C}}\left(T_{\mathcal{C}},-\right)$ induces an equivalence of categories $\mathcal{C} / \operatorname{add} \tau T_{\mathcal{C}} \rightarrow \bmod \operatorname{End}_{\mathcal{C}}\left(T_{\mathcal{C}}\right)$. Let $M_{\gamma}$ be the image of $X_{\gamma}$ under this equivalence.

Building on earlier results in [CC, $\mathrm{CK}, \mathrm{CK} 2, \mathrm{~Pa}, \mathrm{Fu}$ and Keller [FK] proved a cluster expansion formula for the cluster variable $x_{\gamma}$ which is of the form

$$
x_{g}=\sum_{e} \chi\left(G r_{e}\left(M_{\gamma}\right)\right) x(e) y(e),
$$

where the sum is over all dimension vectors $e=\left(e_{1}, e_{2}, \ldots, e_{n}\right), e_{i} \in \mathbb{Z}_{\geq 0}$; where $\operatorname{Gr}_{e}\left(M_{\gamma}\right)$ is the $e$-Grassmannian of $M_{\gamma}$, that is, the variety of submodules of dimension vector $e$; where $\chi$ denotes the Euler-Poincaré characteristic; $x(e)$ is some monomial rational function in the cluster variables $x_{1}, \ldots, x_{n}$, and $y(e)=\prod_{i=1}^{n} y_{i}^{e_{i}}$.

In particular, for any complete $(T, \gamma)$-path $\alpha$, we have $y(e)=y(\alpha)$ if and only if for each $k=1,2, \ldots, n$, the arc $\tau_{i_{k}}$ appears exactly $e_{i_{k}}$ times as a $\gamma$-oriented even arc in $\alpha$. By applying Lemma 6.7 we have proved the following theorem.

Theorem 8.1. Let $M_{\gamma}$ be a rigid indecomposable module of a cluster-tilted algebra of type $A$ or $\tilde{A}$. Then $\chi\left(G r_{e}\left(M_{\gamma}\right)\right)$ is the number of complete $(T, \gamma)$-paths $\alpha$ such that the arc $\tau_{i_{k}}$ appears exactly $e_{i_{k}}$ times as a $\gamma$-oriented even arc in $\alpha$.

When $S$ is a disc this number is 0 or 1 , but if $S$ is an annulus it can be greater than 1, see Example 3.5. In particular, we have the following Corollary.

Corollary 8.2. $\chi\left(G r_{e}\left(M_{\gamma}\right)\right)$ is a non-negative integer.

Remark 8.3. In the case where the cluster-tilted algebra is hereditary, Corollary 8.2 was already proved in [CR. For general cluster-tilted algebras, it is new.

8.2. Projective presentations. Let $T=\left\{\tau_{1}, \tau_{2}, \ldots, \tau_{n+m}\right\}$ be a triangulation of the unpunctured surface $(S, M)$. Let $Q_{T}$ be the quiver defined in section 2.4. Let $A$ be the gentle algebra that the authors in $\mathrm{ABCP}$ associate to the triangulation $T$. The quiver $Q$ of the algebra $A$ is the full subquiver of $Q_{T}$ whose set of vertices is $\{1,2, \ldots, n\}$ and each vertex $j$ corresponds to an interior $\operatorname{arc} \tau_{j}$ of $T$. For each $j=1,2, \ldots, n$, let $P(j)$ denote the indecomposable projective $A$-module at the vertex $j$ of $Q$. Let $M_{\gamma}$ be the indecomposable $A$-module corresponding to the arc $\gamma$. Its dimension vector is given by the crossings of $\gamma$ and the triangulation $T$. Let $I^{-}$and $I^{+}$be the sets defined in section 6.2.

Conjecture 8.4. There is a minimal projective presentation of A-modules of the form

$$
\oplus_{h \in I^{+}} P(h) \rightarrow \oplus_{h \in I^{-}} P(h) \rightarrow M_{\gamma} \rightarrow 0 .
$$

We prove the conjecture in the case where $S$ is a disc or an annulus. With the notation of section 8.1, let

$$
T^{1} \rightarrow T^{0} \stackrel{f}{\rightarrow} X_{\gamma} \rightarrow T^{1}[1]
$$

be a triangle in the cluster category $\mathcal{C}$, where $X_{\gamma}$ is the indecomposable rigid object corresponding to $\gamma, T^{1}, T^{0} \in \operatorname{add} T_{\mathcal{C}}$ and $f$ is a right add $T$-approximation. Denote by $a_{i}^{+}$the multiplicity of the indecomposable $T_{i}$ in $T^{1}$ and denote by $a_{i}^{-}$ the multiplicity of the indecomposable $T_{i}$ in $T^{0}$. In other words, $T^{1}=\oplus_{i=1}^{n} a_{i}^{+} T_{i}$ and $T^{0}=\oplus_{i=1}^{n} a_{i}^{-} T_{i}$. Applying $\operatorname{Hom}_{\mathcal{C}}\left(T_{\mathcal{C}},-\right)$ to the triangle (21), we get a minimal projective presentation

$$
\oplus_{i=1}^{n} a_{i}^{+} P(i) \rightarrow \oplus_{i=1}^{n} a_{i}^{-} P(i) \rightarrow M_{\gamma} \rightarrow 0
$$


in the module category of the cluster-tilted algebra. Following $[\mathrm{Pa}$, we define the index ind $M_{\gamma}$ of $M_{\gamma}$ to be $\left(a_{i}^{+}-a_{i}^{-}\right)_{i} \in \mathbb{Z}^{n}$. Then by [FK, Proposition 5.2], we can relate $g$-vector and index by

$$
g_{\gamma}=- \text { ind } M_{\gamma} \text {, }
$$

where the minus sign is due to our choice of orientation on $(S, M)$. Thus, by Theorem 6.5, the projective presentation (22) is of the form conjectured in 8.4

\section{REFERENCES}

[A] C. Amiot, Cluster categories for algebras of global dimension 2 and quivers with potential, preprint, arXiv:0805.1035

$[\mathrm{ABCP}]$ I. Assem, T. Brüstle, G. Charbonneau-Jodoin, P.G. Plamondon, Gentle algebras arising from surface triangulations, in preparation.

[ABS] I. Assem, T. Brüstle, R. Schiffler, Cluster-tilted algebras as trivial extensions, Bull. London Math. Soc. 40, (2008), 151-162.

[BFZ] A. Berenstein, S. Fomin and A. Zelevinsky, Cluster algebras III: Upper bounds and double Bruhat cells, Duke Math. J.126 (2005), No. 1, 1-52.

[BMRRT] A. Buan, R. Marsh, M. Reineke, I. Reiten and G. Todorov, Tilting theory and cluster combinatorics, Adv. Math. 204 (2006), 572-612 arXiv:math.RT/0402054

[BMR1] A. B. Buan, R. Marsh and I. Reiten, Cluster-tilted algebras, Trans. Amer. Math. Soc.359 (2007), no. 1, 323-332 (electronic).

[BMR2] A. B. Buan, R. Marsh and I. Reiten, Denominators of cluster variables, preprint, arXiv:0710.4335.

[CC] P. Caldero and F. Chapoton, Cluster algebras as Hall algebras of quiver representations, Comment. Math. Helv. 81 (2006), 595-616, arXiv:math.RT/0410187.

[CCS] P. Caldero, F. Chapoton and R. Schiffler, Quivers with relations arising from clusters $\left(A_{n}\right.$ case), Trans. Amer. Math. Soc. 358 (2006), no. 3, 1347-1364, arXiv:math.RT/0401316.

[CCS2] P. Caldero, F. Chapoton and R. Schiffler, Quivers with relations and cluster tilted algebras, Algebr. and Represent. Theory 9, (2006), no. 4, 359-376.

[CK] P. Caldero and B. Keller, From triangulated categories to cluster algebras, to appear in Invent. Math.

[CK2] P. Caldero, B. Keller, From triangulated categories to cluster algebras II, Ann. Sci. École Norm. Sup. (4) 39 (2006), no. 6, 983-1009.

[CR] P. Caldero and M. Reineke, On the quiver Grassmannian in the acyclic case, preprint, arXiv: math.RT/0611074

[CZ] P. Caldero and A. Zelevinsky, Laurent expansions in cluster algebras via quiver representations, Mosc. Math. J. 6 (2006), no. 3, 411-429.

[CP] G. Carroll and G. Price, (unpublished result).

[DWZ] H. Derksen, J. Weyman and A. Zelevinsky, Quivers with potentials and their representations I: Mutations, preprint (2007), arXiv:0704.0649

[FG1] V. Fock and A. Goncharov, Moduli spaces of local systems and higher Teichmüller theory. Publ. Math. Inst. Hautes Études Sci. No. 103 (2006), 1-211.

[FG2] V. Fock and A. Goncharov, Cluster ensembles, quantization and the dilogarithm, preprint (2003) arXiv:math.AG/0311149

[FG3] V. Fock and A. Goncharov, Dual Teichmüller and lamination spaces. Handbook of Teichmüller theory. Vol. I, 647-684, IRMA Lect. Math. Theor. Phys., 11, Eur. Math. Soc., Zürich, 2007.

[FST] S. Fomin, M. Shapiro and D. Thurston, Cluster algebras and triangulated surfaces. Part I: Cluster complexes, preprint (2006) arXiv:math/0608367

[FT] S. Fomin and D. Thurston, Cluster algebras and triangulated surfaces. Part II: Lambda lengths, preprint (2008).

[FZ1] S. Fomin and A. Zelevinsky, Cluster algebras I. Foundations, J. Amer. Math. Soc. 15(2), (2002), 497-529 (electronic), arXiv:math.RT/0104151.

[FZ2] S. Fomin and A. Zelevinsky, Cluster Algebras IV: Coefficients, Comp. Math. 143 (2007), 112-164.

[FZ3] S. Fomin and A. Zelevinsky, (unpublished result). 
[FK] C. Fu and B. Keller, On cluster algebras with coefficients and 2-Calabi-Yau categories, preprint (2008), arXiv:0710.3152

[GSV1] M. Gekhtman, M. Shapiro and A. Vainshtein, Cluster algebras and Poisson geometry. Mosc. Math. J. 3 (2003), no. 3, 899-934, 1199.

[GSV2] M. Gekhtman, M. Shapiro and A. Vainshtein, Cluster algebras and Weil-Petersson forms. Duke Math. J. 127 (2005), no. 2, 291-311.

[LF] D. Labardini-Fragoso, Quivers with potentials associated to triangulated surfaces, preprint (2008) arXiv:0803.1328

[Mo] L. Mosher, Tiling the projective foliation space of a punctured surface. Trans. Amer. Math. Soc. 306 (1988), 1-70.

[MP] G. Musiker and J. Propp, Combinatorial interpretations for rank-two cluster algebras of affine type. Electron. J. Combin. 14 (2007), no. 1, Research Paper 15, 23 pp. (electronic).

[M] G. Musiker, A graph theoretic expansion formula for cluster algebras of classical type, preprint (2007), arXiv:0710.3574

[MS] G. Musiker, R. Schiffler, Cluster expansion formulas and perfect matchings, in preparation. [Pa] Y. Palu, Cluster characters for triangulated 2-Calabi-Yau categories, preprint (2007), arXiv:math/0703540.

[Pr] J. Propp, The combinatorics of frieze patterns and Markoff numbers, preprint (2005), arXiv:math.CO/0511633

[S] R. Schiffler, A cluster expansion formula ( $A_{n}$ case), preprint (2006) arXiv:math.RT/0611956

[ST] R. Schiffler and H. Thomas, On cluster algebras arising from unpunctured surfaces, preprint (2007) arXiv:0712.4131

[SZ] P. Sherman and A. Zelevinsky, Positivity and canonical bases in rank 2 cluster algebras of finite and affine types. Mosc. Math. J. 4 (2004), no. 4, 947-974, 982.

[Z] A. Zelevinsky, Semicanonical basis generators of the cluster algebra of type $A_{1}^{(1)}$. Electron. J. Combin. 14 (2007), no. 1, Note 4, 5 pp. (electronic).

\author{
Ralf Schiffler \\ Department of Mathematics \\ University of Connecticut \\ Storrs, CT 06269-3009 \\ Email: schifflermath.uconn.edu
}

